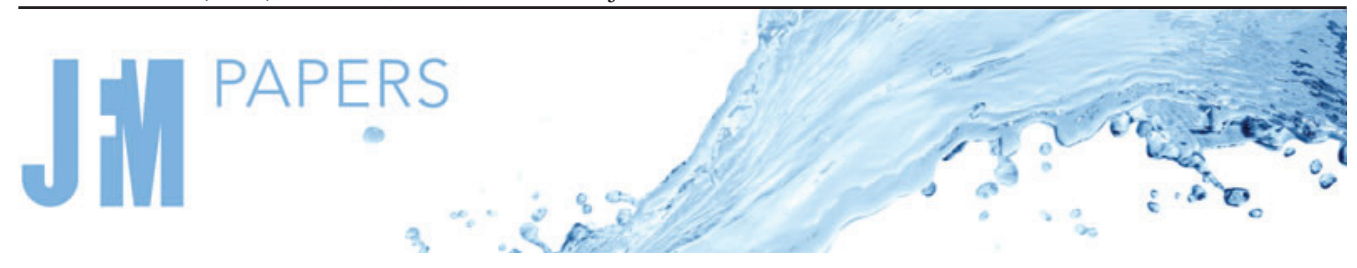

\title{
Numerical investigation of the wake bi-stability behind a notchback Ahmed body
}

\author{
Kan $\mathrm{He}^{1,2,3}$, Guglielmo Minelli ${ }^{4}$, Jiabin Wang ${ }^{4}$, Tianyun Dong ${ }^{4}$, \\ Guangjun Gao ${ }^{1,2,3, \dagger}$ and Siniša Krajnovic ${ }^{4}$ \\ ${ }^{1}$ Key Laboratory of Traffic Safety on the Track of Ministry of Education, School of Traffic \& \\ Transportation Engineering, Central South University, Changsha 410075, PR China \\ ${ }^{2}$ Joint International Research Laboratory of Key Technology for Rail Traffic Safety, Central South \\ University, Changsha 410075, PR China \\ ${ }^{3}$ National \& Local Joint Engineering Research Center of Safety Technology for Rail Vehicle, \\ Changsha 410075, PR China \\ ${ }^{4}$ Division of Fluid Dynamics, Department of Mechanics and Maritime Sciences, Chalmers University of \\ Technology, SE-41296 Gothenburg, Sweden
}

(Received 18 January 2021; revised 17 June 2021; accepted 15 August 2021)

Large-eddy simulations are used to investigate the origin of the wake asymmetry and symmetry behind notchback Ahmed bodies. Two different effective backlight angles, $\beta_{1}=$ $17.8^{\circ}$ and $\beta_{2}=21.0^{\circ}$, are simulated resulting in wake asymmetry and symmetry in flows without external perturbations, in agreement with previous experimental observations. In particular, the asymmetric case presents a bi-stable nature showing, in a random fashion, two stable mirrored states characterized by a left or right asymmetry for long periods. A random switch and several attempts to switch between the bi-stability are observed. The asymmetry of the flow is ascribed to the asymmetric separations and reattachments in the wake. The deflection of the near-wall flow structures behind the slant counteracting the asymmetry drives the wake to be temporarily symmetric, triggering the switching process of the bi-stable wake. The consequence of deflection that forces the flow structure to form on the opposite side of the slant is the decisive factor for a successful switch. Modal analysis applying proper orthogonal decomposition is used for the exploration of the wake dynamics of the bi-stable nature observed.

Key words: wakes

$\dagger$ Email address for correspondence: gjgao@csu.edu.cn

(C) The Author(s), 2021. Published by Cambridge University Press. This is an Open Access article, distributed under the terms of the Creative Commons Attribution licence (http://creativecommons.org/ licenses/by/4.0/), which permits unrestricted re-use, distribution, and reproduction in any medium, provided the original work is properly cited. 


\section{K. He and others}

\section{Introduction}

The aerodynamic performance of ground vehicles is generally dependent on the dynamics and behaviours of the surrounding flow. The time-averaged flow around a symmetric bluff body placed at zero yaw angle normally forms a wake symmetry. This assumption is rooted among researchers performing experimental and numerical results and often used as a criterion for judging the quality of simulations. However, there are observations of asymmetric wakes in cases with symmetric set-up geometries. For example, the mean flow symmetry around a squareback Ahmed body was, in wind tunnel experiments, found to be asymmetric by Grandemange, Cadot \& Gohlke (2012). The squareback Ahmed body used is a modified version that originated from a hatchback Ahmed body, as a typical car-like model, proposed by Ahmed, Ramm \& Faltin (1984). In the asymmetric wake observed by Grandemange et al. (2012), the flow status was found to be sensitive to the Reynolds number in the laminar regime $(310<R e<415)$, and a steady asymmetric state was possible to be maintained for a certain period for $R e=315$. After that, the flow asymmetry was found in a turbulent regime and to switch between two asymmetric states (Grandemange, Gohlke \& Cadot 2013a), showing a wake bi-stability. This phenomenon has experimentally proven to be relevant to several factors that can cause changes in the flow, such as the aspect ratio and ground clearance of the model (Grandemange, Gohlke \& Cadot 2013b), yaw angles (Volpe, Devinant \& Kourta 2015), the depth of a cavity attached to the rear body (Evrard et al. 2016), upstream perturbations in the near-wall region (Barros et al. 2017), rotating wheels (Pavia \& Passmore 2017) and others. For numerical simulations, symmetry breaking has been observed in both the laminar and the turbulent regimes (Östh et al. 2014; Pasquetti \& Peres 2015; Evstafyeva, Morgans \& Dalla Longa 2017; Lucas et al. 2017; Dalla Longa, Evstafyeva \& Morgans 2019) in large-eddy simulations (LES).

Although the flow asymmetry was well presented, the listed works of the literature seem to suffer from a limitation that the squareback body used does not represent a sedan passenger vehicle as its rear is a flat vertical surface without a slanted rear-window surface. A distinction of the wake structures behind the slanted-rear configuration is that, in addition to the notable separation from the shear layer of vertical trailing edges, two C-pillar vortices are shed from both sides of the slant. Moreover, in the near wake behind the squareback end, the momentum in the wall-normal direction caused by the flow separation is not countered by the transport of the wall-tangent momentum and flow reattachment does not occur. In the original hatchback Ahmed body with a slanted rear, the flow separates, and for some angles of slanted surface, it reattaches to the slanted surface (Sims-Williams \& Duncan 2003; Hinterberger, Garcia-Villalba \& Rodi 2004; Krajnović \& Davidson 2005), modifying the wake flow. Attributed to the flow separation and reattachment, another kind of wake bi-stability was observed by Bonnavion et al. (2017). In this full-scale experiment, with a transition yawing angle, two wake states were found to originate from the wake multi-stability caused by the intermittent reattachment on the slanted rear. Moreover, the wake bi-stability behind hatchback Ahmed bodies were observed with small changes of yaw angles (Meile et al. 2016; Rao et al. 2018), showing a phase jump between two wake states under a yaw angle of around $12.5^{\circ}$. The bi-stability behind the hatchback Ahmed body was characterized as the instability of the asymmetric wake separations under yaws. Therefore, the bi-stable wakes involved with the hatchback and the squareback Ahmed bodies are not classified into the same category.

Another kind of Ahmed body with a notchback configuration, representing a sedan passenger vehicle, seems to have higher complexity in the flow and can form wake 
bi-stability under zero yaws (Sims-Williams, Marwood \& Sprot 2011). This model is a simplified version of a sedan vehicle representing both the rear slanted window and rear part of the vehicle, allowing the flow reattachment to the rear part. A description of the flow around a notchback geometry was provided by Carr (1974), who found that the reattachment of the flow on the rear part occurs with effective backlight angle $\beta<35^{\circ}$. The first experimental observation of the asymmetry in the time-averaged flow around a notchback car was reported by Cogotti (1986). This experiment identified that a variation of the yaw angle from 0 to $1^{\circ}$ results in a switch of two mirrored asymmetric wake states. A secondary observation from Cogotti (1986) showed that the change of the asymmetric status was a rather slow process, where the asymmetry lasted for several minutes on one side. Furthermore, using surface flow visualization and laser velocimetry, Jenkins (2000) sketched the flow structures around the rear of a notchback body, which had rounded edges being a more realistic representation of a simplified car. Although the asymmetric flow was not reported, the sketch of the time-averaged flow was new in the study of Jenkins (2000) and different from those of Carr (1974) and Cogotti (1986). In particular, vortices behind the backlight and close to the decklid were found to move toward the central plane. Afterwards, Gilhome, Saunders \& Sheridan (2001) used surface flow visualization, critical-point theory and smoke visualization to describe a more complex flow topology around the rear end of two sedan-type vehicles. In addition to C-pillar trailing vortices and the recirculation region on the slanted surface, large hairpin vortices, recirculation vortices and a pair of streamwise vortices resulting from the hairpin vortices were visualized. The new flow features were found to originate in the recirculating region on the slanted surface, and their existence was deduced based on experimental evidence. Furthermore, the flow asymmetry was visualized with particle image velocimetry around a notchback saloon (Lawson, Garry \& Faucompret 2007), indicating that flow asymmetry in the backlight region appears to be more sensitive to a small change of yaw angles or acceleration of the inlet speed, rather than a traditional Reynolds-number-related mechanism. With regard to the flow asymmetries over the rear of different notchback models, an overview of previous investigations is presented by Gaylard, Howell \& Garry (2007), showing that further studies are needed to explain the underlying flow physics causing asymmetric wakes. For instance, as mentioned before, Sims-Williams et al. (2011) used several different experimental techniques including surface-flow visualization and particle image velocimetry to study the flow asymmetry of 16 different notchback models. That study showed a clear dependency of the flow asymmetry on the angle of the slanted surface of the notchback Ahmed body. Therefore, although the shape of the fore and the middle body is the same as that of the model investigated by Grandemange et al. $(2012,2013 a$, $2013 b$ ), the variability in the rear configuration is sure to result in different asymmetric flows. However, the underlying flow mechanism causing the asymmetry for a notchback body is formed by the wake structures, involving the interaction of the central vortex shed from the shear layer of the upper slant and the C-pillar vortices. This motivates the present LES investigation considering the geometries with disparate slanted angles proposed by Sims-Williams et al. (2011), to explore the mechanisms of the wake asymmetry behind a notchback Ahmed body from the dynamics of flow structures.

Moreover, although switches between two asymmetric mirrored states of wake bi-stability have been experimentally observed for squareback bluff bodies (Grandemange et al. 2013a, 2013b; Volpe et al. 2015; Haffner et al. 2020; Pavia et al. 2020), the random switch in the notchback case has remained unexplained. It is worth noting that random switches between bi-stable wakes behind squareback Ahmed bodies were predicted by 


\section{K. He and others}

Östh et al. (2014) and Dalla Longa et al. (2019) with the LES approach. Those two studies numerically repeated the wake bi-stability observed by Grandemange et al. (2013b), and proved feasible for predicting the switch of the wake asymmetry behind notchback Ahmed bodies. Although requiring the simulation of a long physical time scale for capturing the shifting process of bi-stability, a random switch of the bi-stable flow is observed during the LES in the present work. As far as the authors know, this is the first time that a switch between bi-stable states of a notchback configuration has been simulated. Furthermore, several attempts to switch between the two asymmetric mirrored states during the simulation are detected. Therefore, the possibility of substantiating the reason for triggering flow to switch is provided by comparing the successful and failed switches.

The purpose of the present work is to elucidate the mechanism of the wake asymmetry behind notchback configurations with a specific focus on the random switch observed in the bi-stable wake. The bi-stable nature observed for the notchback body is fundamentally different from that for the squareback type (Grandemange et al. 2012, 2013a, 2013b; Pavia et al. 2020), due to the distinguishable shedding vortices from the slant and the flow reattachment to the deck. The results of this work help to explore the fluid mechanisms of the wake bi-stability behind a notchback bluff body with the interaction of the wake structures.

The remainder of the paper is organized as follows. Section 2 presents a description of the geometry, domain, numerical method, post-processing set-up and modal analysis. In $\S 3$, the results are analysed with the focus on the flow features around two notchback Ahmed bodies. The switching process of the bi-stable wake is investigated, with the flow structures and modal analysis applying proper orthogonal decomposition (POD). Conclusions follow in $\S 4$. The grid independence examination is presented in Appendix A. A brief description of two asymmetric mirrored states obtained using several well-resolved meshes is presented in Appendix B.

\section{Methodology}

\subsection{Geometry and boundary conditions}

Two notchback Ahmed bodies used for numerical simulations are shown in figure 1 . The wake is expected to be asymmetric behind Model 1 and symmetric behind Model 2 following the experimental study by Sims-Williams et al. (2011). The same dimensions of the two models are expressed in the height of the model, $H=0.096 \mathrm{~m}$. The width of the model is $W=1.35 H$, the model length is $L=3.82 H$, the deck length is $L_{D}=0.469 H$ and the deck height is $H_{D}=0.687 \mathrm{H}$. The clearance between the ground and the bottom of the model is $H_{C}=0.21 H$. The only difference between the two models is in the effective backlight angles, $\beta_{1}=17.8^{\circ}$ of Model 1 and $\beta_{2}=21.0^{\circ}$ of Model 2, giving the roof lengths $L_{S 1}=2.847 \mathrm{H}$ and $L_{S 2}=3.006 \mathrm{H}$. The origin of the coordinate is set at the middle body in the $x$ direction, the middle section of the model in the $y$ direction and the bottom body in the $z$ direction.

The model is centred in a digital tunnel as illustrated in figure 2. The cross-sectional width of the tunnel domain is $W_{T}=11.46 \mathrm{H}$ and the tunnel height is $H_{T}=5.73 \mathrm{H}$. The inlet is set at a distance of $D_{\text {in }}=8 \mathrm{H}$ upstream of the model and the outlet is located at $D_{\text {out }}=19 H$ downstream of the model. The Reynolds number, $R e=5 \times 10^{4}$, is based on the model height and a uniform inlet velocity profile $U_{\text {inf }}=7.864 \mathrm{~m} \mathrm{~s}^{-1}$. The pressure outlet with a constant pressure of $0 \mathrm{~Pa}$ is used at the downstream boundary. The lateral and roof surfaces are set as symmetry planes. The no-slip wall boundary condition is applied 
(a)

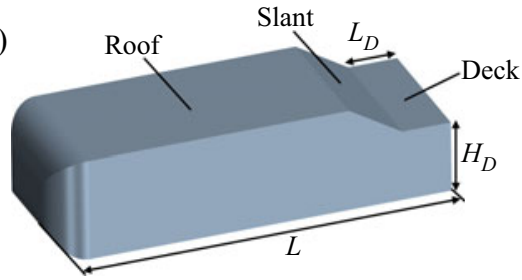

(c)

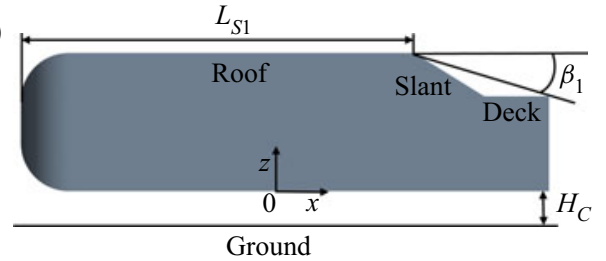

(b)

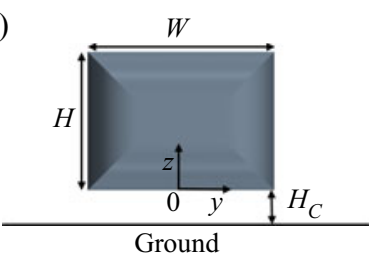

$(d)$

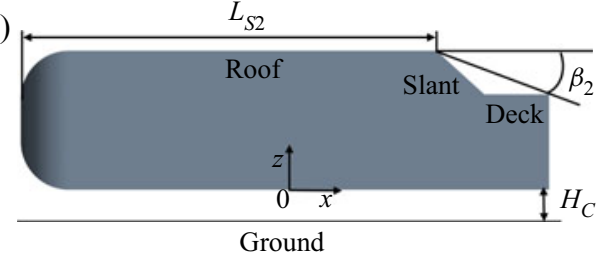

Figure 1. Geometric model: (a) aerial view of Model 1; $(b)$ front view of the model; $(c)$ side view of Model 1; $(d)$ side view of Model 2.

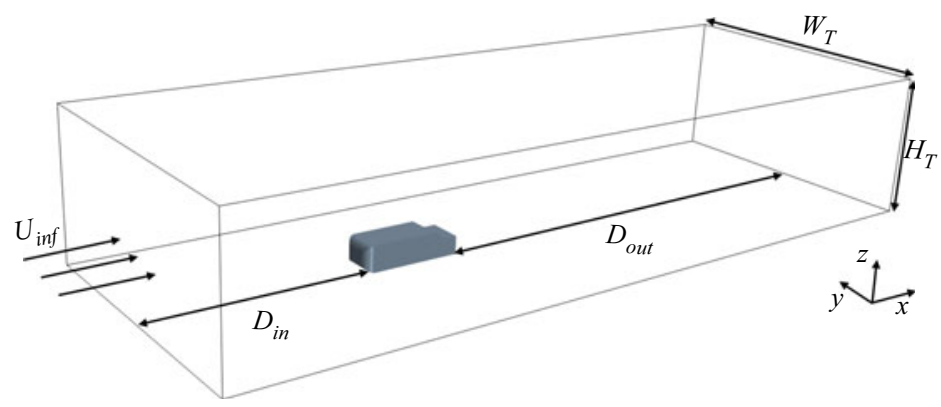

Figure 2. The flow domain for simulations.

on the surface of the models, and the ground of the domain is treated as a moving wall using the same velocity as $U_{\text {inf }}$.

\subsection{Numerical method and details}

The governing LES equations solved with a commercial finite volume solver, Star CCM+ 2019.2, are the incompressible Navier-Stokes and the continuity equations filtered with the implicit spatial filter of characteristic width $\Delta$. The filter width, $\Delta$, is defined as $\Delta=\left(\Delta_{1} \Delta_{2} \Delta_{3}\right)^{1 / 3}$, where $\Delta_{i}$ are the computational cell sizes in three coordinate directions. The wall-adapting local eddy-viscosity (WALE) model proposed by Nicoud \& Ducros (1999), which has been extensively tested and applied to predict flows around the hatchback (Aljure et al. 2014), the squareback (Dalla Longa et al. 2019) and the notchback (He et al. 2021) Ahmed bodies, is used in this work. This subgrid-scale (SGS) model is based on the square of the velocity gradient tensor and accounts for the effects of the strain and rotation rates. The subgrid eddy viscosity in the WALE model is modelled as

$$
\mu_{t}=\rho\left(C_{w} \Delta\right)^{2} \frac{\left(S_{i j}^{d} S_{i j}^{d}\right)^{3 / 2}}{\left(\tilde{S}_{i j} \tilde{S}_{i j}\right)^{5 / 2}+\left(S_{i j}^{d} S_{i j}^{d}\right)^{5 / 4}},
$$




\section{K. He and others}

where $C_{w}$ is a constant, here $C_{w}=0.544$. Tensor $\tilde{S}$ is the filtered rate of strain tensor and $S_{i j}^{d}$ is the traceless symmetric part of the square of the velocity gradient tensor $g_{i j}$, defined as

$$
S_{i j}^{d}=\frac{1}{2}\left(\tilde{g}_{i j}^{2}+\tilde{g}_{j i}^{2}\right)-\frac{1}{3} \delta_{i j} \tilde{g}_{k k}^{2},
$$

where $g_{i j}$ denotes $\partial u_{i} / \partial x_{j}$.

Convective fluxes are approximated by a blend of $98 \%$ central difference scheme of second-order accuracy and $2 \%$ first-order upwind scheme. The time integration is done using the second-order-accurate three-level time Euler scheme. The non-dimensional time step $\mathrm{d} t^{*}=\Delta t_{S} U_{\text {inf }} / H=3.44 \times 10^{-3}$, giving a Courant-Friedrichs-Lewy number lower than 1 in over $99 \%$ of the cells during all time steps, including the simulation applying the fine mesh presented in Appendix A. Reynolds-averaged Navier-Stokes simulations are first conducted as initial conditions for all LES. Then, the flow is sampled after at least the initial $t^{*}=t U_{\text {inf }} / H=62$. The sampling duration of the simulation for Model 1 is $t^{*}=930$ corresponding to 30 flow-through passages through the domain and that for Model 2 is $t^{*}=124$.

\subsection{Measurement set-up}

The aerodynamic drag force $F_{d}$ is the component of the aerodynamic load in the $x$ direction associated with the coordinate system defined in figure 1 . The aerodynamic coefficient of the drag, $C_{d}$, is defined as

$$
C_{d}=\frac{F_{d}}{\frac{1}{2} \rho A U_{\text {inf }}^{2}},
$$

where $\rho$ is the density of the fluid, $A$ is the projected area of the frontal model surface in the $x$ direction, as shown in figure $1(b)$, and $U_{\text {inf }}$ is the inlet velocity of the free stream.

The velocity components in the $x, y$ and $z$ directions are $U_{x}, U_{y}$ and $U_{z}$, respectively. The associated streamwise, spanwise and vertical velocity components, $u, v$ and $w$, are defined as

$$
u=\frac{U_{x}}{U_{i n f}}, \quad v=\frac{U_{y}}{U_{i n f}}, \quad w=\frac{U_{z}}{U_{i n f}} .
$$

The coefficient of pressure $C_{p}$ is defined as

$$
C_{p}=\frac{p-p_{\infty}}{\frac{1}{2} \rho U_{i n f}^{2}},
$$

where $p$ is the pressure and $p_{\infty}$ is the pressure in the upstream far field.

The base pressure $C_{p B i}$ on each surface of the rear body is defined as

$$
C_{p B i}=\frac{1}{A_{i}} \int C_{p} \cdot \mathrm{d} A_{i},
$$

where $i \in\{s, d, b\}$ stands for the slant, the deck and the back wall, respectively, and $A_{i}$ is the area of each surface.

Pressure probes are in the same way set at the two rear bodies, e.g. for Model 1 shown in figure 3. Two monitoring points, symmetrically distributed on the left and right sides, are arranged on each surface of the slant, the deck and the back wall. The distance between the central line and the corresponding side point is $d y=0.417 \mathrm{H}$. The points on the slant are 


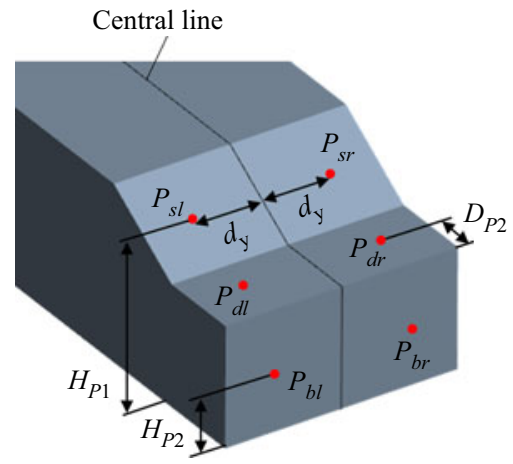

Figure 3. Pressure monitoring points on the rear surface of Model 1.

located at $H_{P 1}=0.844 H$ above the bottom of the model (at half-height of the slant). The points on the deck are at $D_{P 2}=0.5 L_{D}$ (half-length of the deck) from the trailing edge of the deck. The points on the back wall are at $H_{P 2}=0.5 H_{D}$ (half-height of the deck) above the bottom of the model. The pressure is sampled at the last iteration of each time step during the simulation.

The extent of the asymmetry in the wake is quantified by the spanwise base pressure gradients, defined as

$$
\frac{\partial C_{p i}}{\partial y}=\frac{C_{p}\left(P_{i r}\right)-C_{p}\left(P_{i l}\right)}{2 d y},
$$

where $i \in\{s, d, b\}$ stands for the slant, the deck and the back wall, respectively, and $C_{p}()$ represents the sampled pressure coefficient on each monitoring point. By the definition of the base pressure gradients, a higher absolute value of $\partial C_{p i} / \partial y$ indicates a higher degree of wake asymmetry. On the contrary, $\partial C_{p i} / \partial y$ fluctuating around 0 suggests wake symmetry.

\subsection{Description of modal analysis}

Fast Fourier transform (FFT) and POD are performed for modal analysis of the wake flow. The POD approach provides an in-depth understanding of flow structures in terms of both energy contents and characteristic frequencies. As originally proposed by Lumley (1970), and later introduced with the method of snapshots by Sirovich (1987), this method is based on the energy ranking of orthogonal structures predicted from a correlation matrix of the snapshots. A singular-value decomposition approach is used to conduct the POD analysis. The time step between snapshots is $\Delta t=4.2 \times 10^{-4} \mathrm{~s}, 10$ times $\Delta t_{s}$ in simulations. Thus, the highest frequency considered in the modal analysis (the Nyquist frequency) is $1190 \mathrm{~Hz}$. The lowest frequency captured is limited by the snapshot total sampling time to $13 \mathrm{~Hz}$ (the snapshots are collected more than $t^{*}=62$ and at least ten periods should be captured for a reliable frequency evaluation). These two frequencies give a reliable real frequency range between 13 and $1190 \mathrm{~Hz}\left(0.16<S t_{H}<14.52\right)$. The non-dimensional frequency $S t_{H}$ is the Strouhal number normalized with the free-stream velocity and the height of the model. The POD method has been successfully used in previously published works for flows of bluff bodies by Thacker et al. (2013), Östh et al. (2014), Minelli et al. (2016) and others. In the present work, the modal analysis used for the characterization of the wake dynamics is based on the energy content, identifying flow structures contributing to the asymmetry or symmetry of the wake. In particular, the POD algorithm is applied with a zonal approach for analysing the frequency content of the considered field portion. 


\section{K. He and others}

\section{Analysis and discussion}

Flow features around Model 1 and Model 2 are analysed in this section. It is divided into two subsections. In $\S 3.1$, the results of the wake asymmetry behind Model 1 and the wake symmetry behind Model 2 are presented following the experimental investigation by Sims-Williams et al. (2011). In $\S 3.2$, the switch observed between the bi-stable wake is explored by comparing the successful and the failed switching periods. Modal analysis applying POD is presented in both subsections with the aim of exploring the dynamics of the wake structures.

\subsection{Asymmetric and symmetric flows}

The wake asymmetry behind Model 1 and the symmetry behind Model 2 are observed in the LES. Numerical accuracy based on the fine, the medium and the coarse meshes is established by a grid-independence examination presented in Appendix A. The time history of the base pressure gradients on the slant surface during the entire simulation for Model 1 is plotted in figure 4(a). Two stable asymmetric mirrored states observed during the simulation are expressed by two representative periods, $S_{a}$ during $t^{*} \in[0,62]$ and $S_{b}$ during $t^{*} \in[558,620]$, respectively shown in figures $4(b)$ and $4(c)$ by expanding the time scale. The two mirrored states establishing bi-stability of the wake are distinguished by the base pressure gradients that fluctuate with positive values during $S_{a}$ but with negative values during $S_{b}$. The pressure gradients during $S_{a}$ showing lower absolute values than $S_{b}$ are not linked to the transient period at the beginning of the computation, since the data are sampled after two flow-through passages through the domain ensuring a full flow development. Performing multiple simulations for capturing the switch of bi-stability, although the meshes are well resolved, the direction of the asymmetry remains sensitive to the mesh (see details in Appendix B). Therefore, the two states of bi-stability can be duplicated in the present LES, and the lower values of $S_{a}$ are attributed to the turbulent fluctuation in the flow evolution. In contrast to the asymmetry behind Model 1 , the surrounding flow of Model 2 is significantly different in that the pressure gradients fluctuate around 0 indicating the symmetry. The time period $t^{*} \in[62,124]$, denoted $S_{c}$, is selected from the flow around Model 2 as the representation of the stable symmetric state (see figure $4 d$ ).

To compare the asymmetric and symmetric wakes, mean flows around Model 1 (during $S_{b}$ ) and Model 2 (during $S_{c}$ ) are presented. Figure 5 shows the distribution of the mean surface pressure (left-hand column) and the probability density function (PDF) of the base pressure gradients against the base pressure. For Model 1, the right-hand side of the slant shows negative pressure. On the right-hand side of the deck, a positive pressure region can be found (see figure $5 a$ ). In figure $5(b)$, however, the symmetry of the surface pressure is indicated by showing equivalent distributions on the two sides. The flow states can be quantitatively assessed by the base pressure gradients. As shown in figure 5(c), the PDF indicates that the values of the base pressure gradients are concentrated in the negative or positive region as a result of the asymmetric flow patterns in the wake. On the other hand, figure $5(d)$ demonstrates the symmetry of the wake behind Model 2, showing the concentration of the pressure gradients around 0 . Moreover, focusing on the base pressure, particularly $C_{p B s}$ and $C_{p B d}$ on the slant and deck, the values are lower in the case of Model 2. The base pressure contributing to the difference in the aerodynamic drag between the two models is discussed later in this section.

The distribution of the surface pressure on the rear body is associated with the flow structures in the wake. Visualizing the iso-surface of the mean pressure, the averaged 

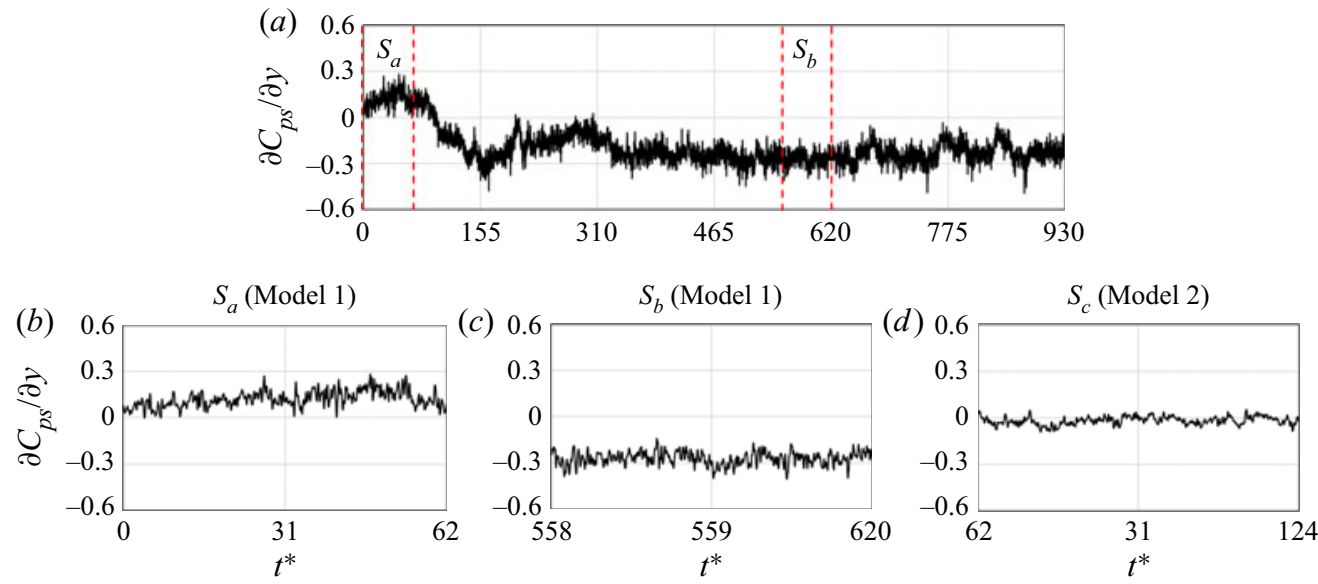

Figure 4. Time history of the base pressure gradients on the slant: $(a)$ the entire simulation for Model $1 ;(b)$ a stable asymmetric state for Model 1 during $S_{a} ;(c)$ an opposite stable asymmetric state for Model 1 during $S_{b}$; $(d)$ a stable symmetric state for Model 2 during $S_{c}$.

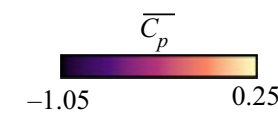

(a)

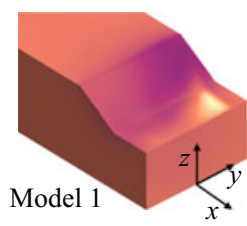

(b)

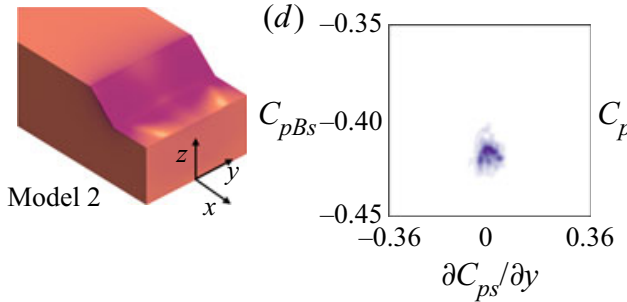

(c)

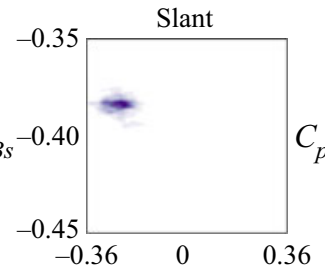

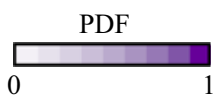
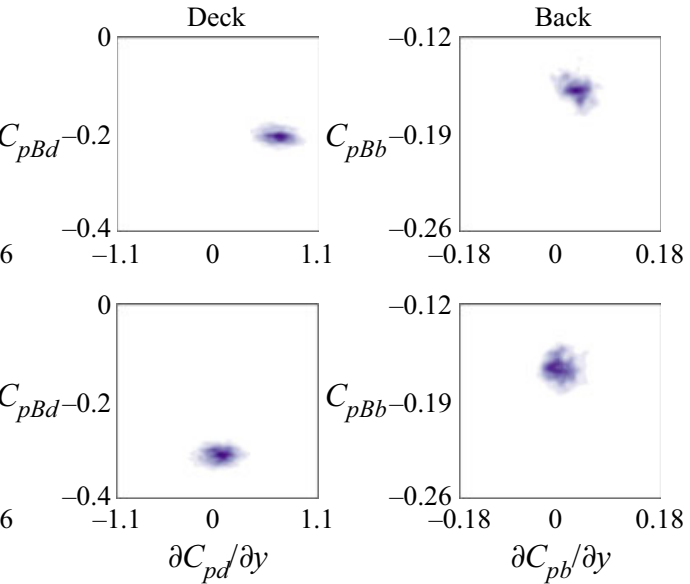

Figure 5. The mean surface pressure distributed on the rear body: (a) Model 1, during $S_{b}$; (b) Model 2, during $S_{c}$. The PDFs of the base pressure gradients against the base pressure on the slant, the deck and the back wall: (c) Model 1, during $S_{b} ;(d)$ Model 2, during $S_{c}$.

flow structure $V_{c}$ behind the slant of the two models is presented in figure $6(a, b)$. During $S_{b}$ and $S_{c}$, the placement of $V_{c}$ is found to be different between the two models. While its extension for Model 1 is limited to the region near the upper-right corner, Model 2 produces a structure stretching between the two upper corners (see figure $6 b$ ). The shape of $V_{c}$ in the two cases is in agreement with the negative pressure on the slant surface observed in figure $5(a, b)$.

Furthermore, the negative pressure inside $V_{c}$ is attributed to the separation from the upper slant, as illustrated by the near-wall traces in figure $6(c, d)$. The flow separating from the upper slant constitutes two counter-rotating vortices, $V_{c l}$ on the left-hand side and $V_{c r}$ on the right-hand side. During $S_{b}$, the region of $V_{c r}$ is larger, forming a recirculation bubble 


\section{K. He and others}
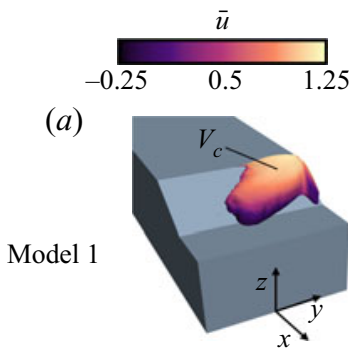

(c)
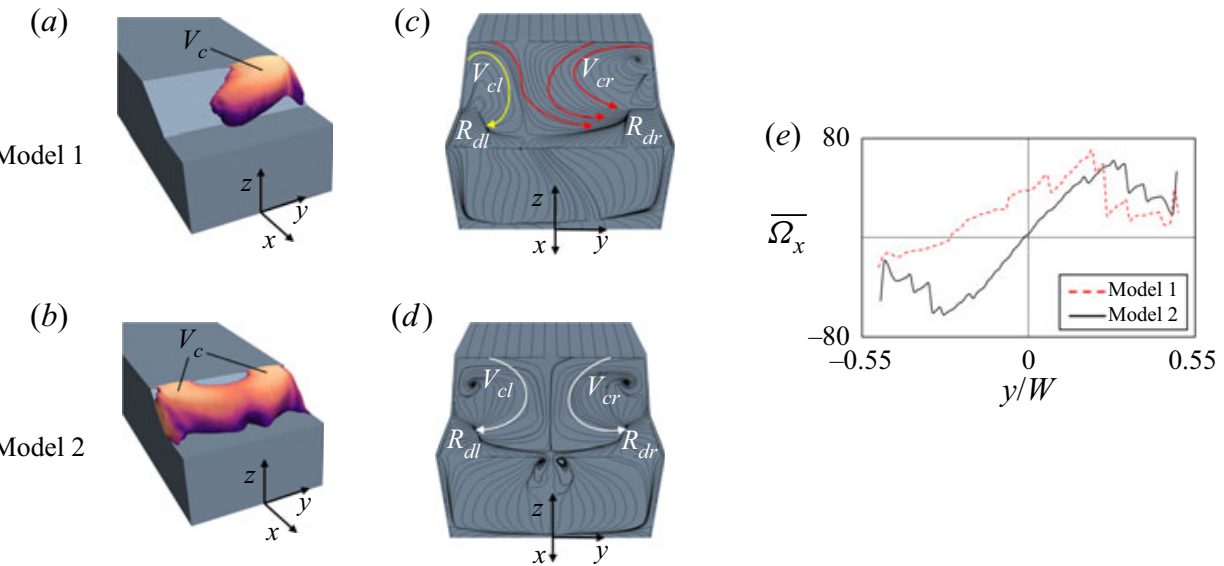

Figure 6. Flow topology visualized by iso-surfaces of the mean pressure at $\overline{C_{p}}=-0.8$, coloured by the mean streamwise velocity, $\bar{u}$ : (a) Model 1, during $S_{b} ;(b)$ Model 2, during $S_{c}$. Averaged near-wall flow traces on the rear surface: $(c)$ Model 1, during $S_{b} ;(d)$ Model 2, during $S_{c}$. (e) Profiles of the streamwise vorticity, $\overline{\Omega_{x}}$. The horizontal probe line is located at the near-wall region, $0.01 \mathrm{H}$ away behind the roof's trailing edge.

at the upper-right of the slant resulting in the asymmetric formation of $V_{c}$. Meanwhile, as indicated by the red arrows in figure $6(c)$, the flow separating from the upper part of the slant reattaches to the right-hand side of the deck, resulting in the reattachment $R_{d r}$. This explains the positive pressure on the right-hand side of the deck as shown in figure $5(a)$. Another reattachment, $R_{d l}$, with a shorter reattaching length is found on the left-hand side of the deck. However, $R_{d l}$ is driven by the flow separating from the left C-pillar, as indicated by the yellow arrow in figure $6(c)$. Shown in figure $6(d)$, the recirculation bubbles shaped by the two shedding vortices can be observed at the two sides during $S_{c}$, forming the symmetry of $V_{c}$ on the slant. As illustrated by the white arrows, the flow separating from the upper slant reattaches to both sides of the deck. The two reattachments, $R_{d l}$ and $R_{d r}$, are in agreement with two symmetric regions of positive pressure on the deck presented in figure $5(b)$.

The extent of the asymmetry characterized by the separation on the slant is quantitated by the horizontal profile illustrating the streamwise vorticity, $\overline{\Omega_{x}}$, behind the near-wall region of the roof's trailing edge (see figure $6 e$ ). The vorticity is normalized by the free-stream velocity and the height of the model. Vorticity $\overline{\Omega_{x}}$ for Model 1 is found to be positive for most of the lateral extension, indicating the asymmetry of the separation from the slanted surface. In the case of Model $2, \overline{\Omega_{x}}$ is found to be negative on the left-hand side but positive on the right-hand side, as a result of the symmetric separation on the slant.

The identification of the positions of flow separations and reattachments visualized by the spanwise vorticity, $\overline{\Omega_{y}}$, and the streamlines projected on the planes in the lateral direction are presented in figure 7 . For quantitative analysis, the separation angle, $\theta$, is defined as the angle between the streamwise direction ( $x$ coordinate) and the line linking the separation point and the position on the contour at $\overline{\Omega_{y}}=3.7$ with the maximum $x$ coordinate. The reattachment length, $L_{R}$, is defined by the distance between the positive-negative transition point of near wall $\overline{\Omega_{y}}$ and the trailing edge of the deck. As indicated by the streamlines and distribution of $\overline{\Omega_{y}}$, the flow first separates from the shear 


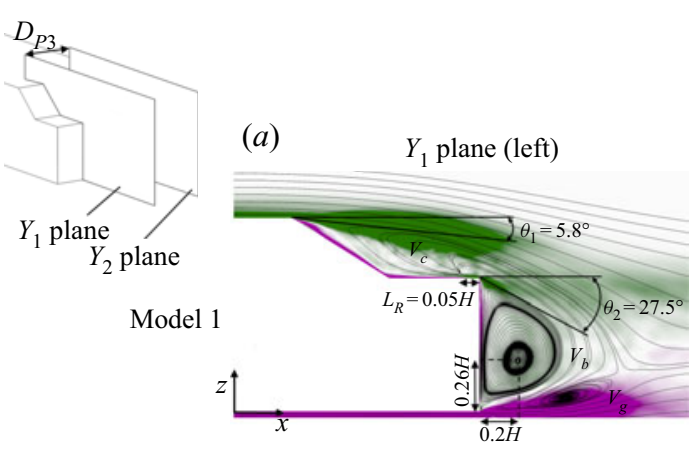

(c)

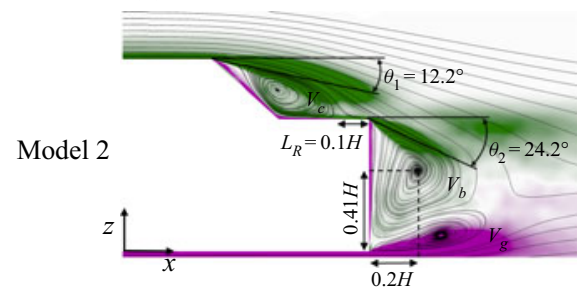

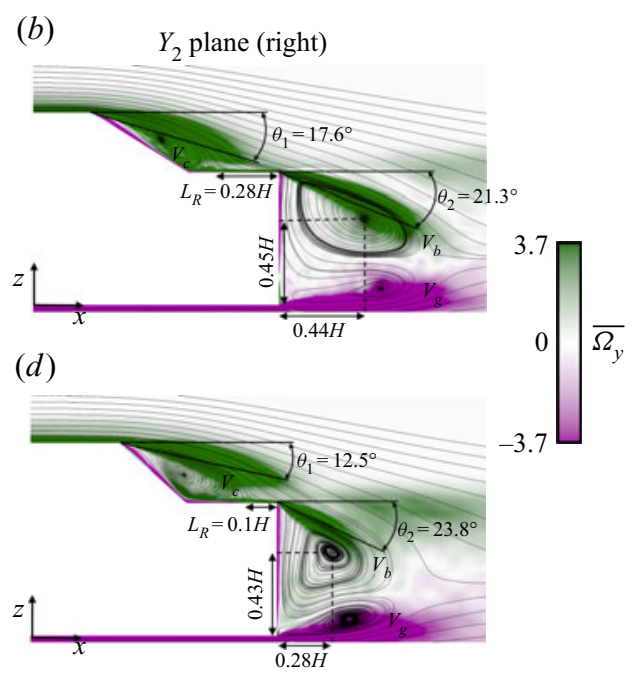

Figure 7. Mean streamlines and the spanwise vorticity, $\overline{\Omega_{y}}$, on the left $Y_{1}$ plane $(a, c)$ and the right $Y_{2}$ plane $(b, d)$. The distance between $Y_{1}$ and $Y_{2}$ is $D_{P 3}=0.5 \mathrm{~W}$ (half-width of the model). Model 1, during $S_{b}:(a)$ on the $Y_{1}$ plane; $(b)$ on the $Y_{2}$ plane. Model 2, during $S_{c}:(c)$ on the $Y_{1}$ plane; $(d)$ on the $Y_{2}$ plane.

layer near the roof's trailing edge. Therefore, a recirculation bubble associated with $V_{c}$ forms above the slant. A second separation from the near-wall region of the deck produces a recirculation bubble, $V_{b}$, behind the back wall. Near the ground, the vortex shedding from the shear layer of the underbody generates another recirculation region $V_{g}$.

Figure $7(a, b)$ showing the two section planes for Model 1 indicates that the separation angle $\theta_{1}$ on the left-hand side $\left(5.8^{\circ}\right)$ is smaller than that on the right-hand side $\left(17.6^{\circ}\right)$. The separations at two different angles influence the downstream flow. The flow coming from the upper part of the slant, on the left-hand side of the body, results in a large slant angle $\theta_{2}\left(27.5^{\circ}\right)$ of the separation from the deck. As a result, the recirculation bubble $V_{b}$ is attached to the vertical base wall. Although the flow on the left-hand side moves over the deck shown by the streamlines, $L_{R}$ with a shorter reattaching length is observed on the deck. As previously discussed, this reattachment is formed by the flow separating from the left C-pillar (see figure $6 c$ ). However, on the right-hand side, the flow coming from the upper part of the slant reattaches to the deck, resulting in a smaller $\theta_{2}\left(21.3^{\circ}\right)$. Thus, $V_{b}$ extends further on the left-hand side. Comparing the core position of $V_{b}$ on the two sides identifies the downstream asymmetry behind the back wall. As above, the wake asymmetry behind Model 1 is characterized by asymmetric wake separations and reattachments.

On the other hand, during $S_{c}$, symmetric separations are found behind the slant of Model 2, as shown in figure $7(c, d)$. The flow separating for the angle, $\theta_{1}=12.2^{\circ}$ on the left and $\theta_{1}=12.5^{\circ}$ on the right, shapes the identical length of $L_{R}$ on both sides of the deck. Besides, the second separation from the deck, with $\theta_{2}=24.2^{\circ}$ on the left and $\theta_{2}=23.8^{\circ}$ on the right, results in comparable recirculations behind the back wall on the two sides. Therefore, the placement of the separations and the reattachments confirms the symmetry of the wake behind Model 2.

For the explanation of the flow separating from the shear layer of the upper slant, schematic representations of streamlines behind the two models are presented in figure 8 . 


\section{K. He and others}

(a)

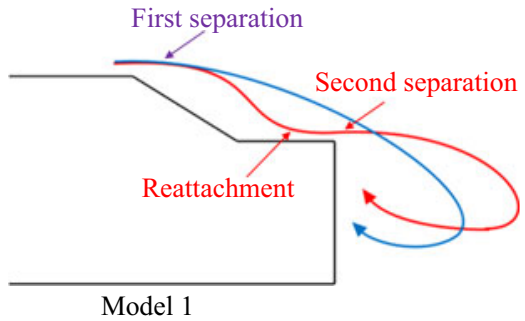

(b)

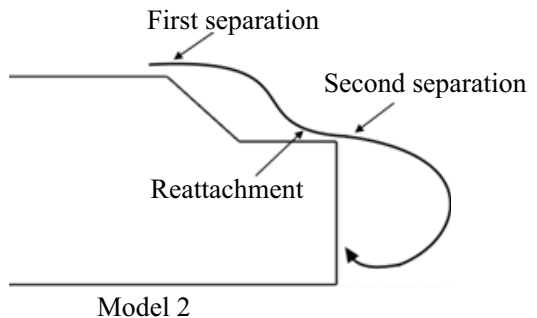

Figure 8. Sketch of the streamlines describing the flow separating from the shear layer of the upper slant: (a) Model 1, during $S_{b} ;(b)$ Model 2, during $S_{c}$.

Figure 8(a) represents the case of wake asymmetry behind Model 1, illustrating that, on one side, the flow separating for a lager angle from the upper slant reattaches to the deck (shown in red). Thereby, the downstream flow separates for a small angle from the deck, forming a recirculation bubble away from the back wall. On the opposite side, the flow separating for a small angle from the upper slant rejects reattaching to the deck, moving downstream over the deck (shown in blue). For Model 2, the wake symmetry is illustrated in figure $8(b)$, indicating consistent separations from the upper slant on both sides, followed by symmetric downstream reattachment and the second separation.

The recirculation bubble formed by the second separation from the deck influences the downstream flow structures behind the back wall. In figure $9(a, b)$, two reverse recirculations, $V_{b s l}$ and $V_{b s r}$, are formed by the vortices shedding from two side edges of the back wall. Another two counter-rotating recirculation bubbles, $V_{b l}$ and $V_{b r}$, are found further away from the lateral edges of the body. Here, $V_{b l}$ and $V_{b r}$ are the leftand right-hand parts of $V_{b}$ generated by the separation from the deck. During $S_{b}$, for Model 1, these vortices are asymmetric as $V_{b l}$ and $V_{b r}$ deflect to the left. Thus $V_{b s r}$ on the right-hand side develops into a larger structure. The corresponding vortices for Model 2 (during $S_{c}$ ) are found to be symmetric around the symmetry line of the body (figure $9 b$ ). The pressure distribution behind the back wall is dependent on the recirculation bubbles. In figure $9(c-f)$, visualizing the iso-surface of negative mean pressure shapes a toroidal vortex behind the rear model. The toroidal vortex is known as an important structure in the wake of bluff bodies and has already been repeatedly observed by Krajnović \& Davidson $(2003,2005)$ and Roumeas, Gillieron \& Kourta (2009). The form of the toroidal vortex follows the recirculation bubbles behind the back wall. Once $V_{b}$ extends further downstream on the right-hand side (see figure $7 b$ ), the right-hand part of toroidal vortex tilts backwards, as shown in figure $9(c)$. On the other hand, when the recirculations behind the back wall are symmetric (see figure $7 c, d$ ), the toroidal vortex tilts on both sides, as shown in figure $9(d)$.

By visualization of the mean iso-surface of the $Q$-criterion, the statistically averaged flow structures behind the slant are presented in figure $10(a-d)$. The non-dimensional $Q^{*}$ is normalized by $Q^{*}=Q\left(H / U_{i n f}\right)^{2}$. Here, the left C-pillar vortex $V_{l}$ consists of two subvortices, $V_{l 1}$ and $V_{l 2}$, separating from the left-hand edges of the slant and the deck. On the right-hand side, $V_{r}$ consists of $V_{r 1}$ and $V_{r 2}$ separating from the right-hand edges of the slant and the deck. Meanwhile, $V_{c}$ formed by separations from the shear layer of the roof's trailing edge is found on the slant. Moreover, fragmented structures $V_{f l}$ and $V_{f r}$ are formed on the deck, showing opposite spanwise velocity.

During $S_{c}$, figure $10(a)$ suggests that $V_{c}$ primarily forms on the right-hand side of the upper slant, confirming the flow structure visualized by pressure iso-surfaces in 


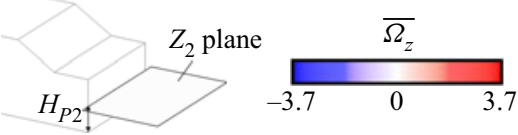

(a)

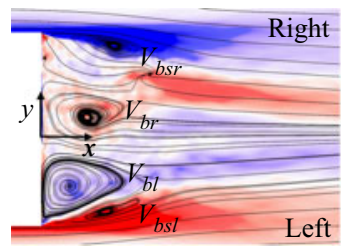

(b)

Model 2

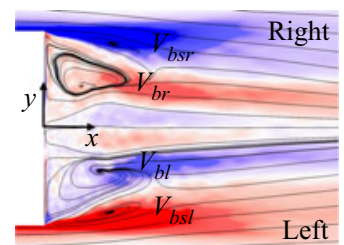

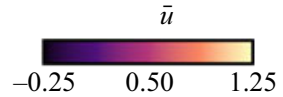

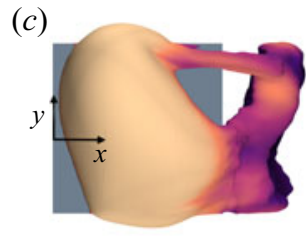

(e)

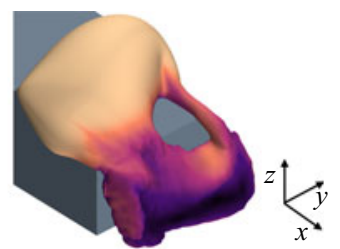

(d)

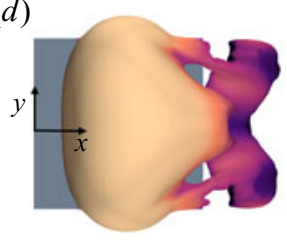

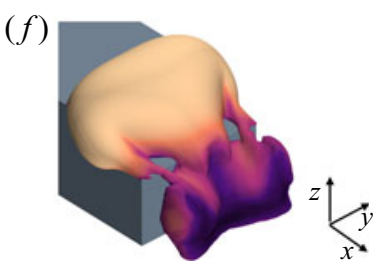

Figure 9. Mean streamlines and vertical vorticity, $\overline{\Omega_{z}}$, on the $Z_{2}$ plane located at $H_{P 2}=0.5 H_{D}$ (half-height of the deck) above the bottom model: (a) Model 1, during $S_{b} ;(b)$ Model 2, during $S_{c}$. Flow structures visualized by iso-surfaces of mean pressure at $\overline{C_{p}}=-0.4$. Top view: (c) Model 1, during $S_{b} ;(d)$ Model 2 , during $S_{c}$. Perspective view: $(e)$ Model 1, during $S_{b} ;(f)$ Model 2, during $S_{c}$.

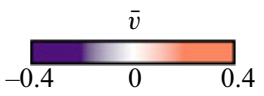

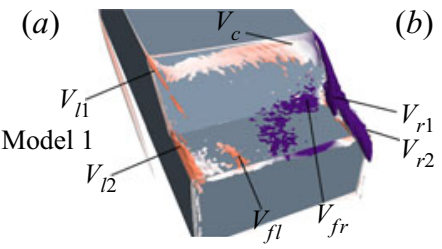

$Q^{*}=14.9$

(c)

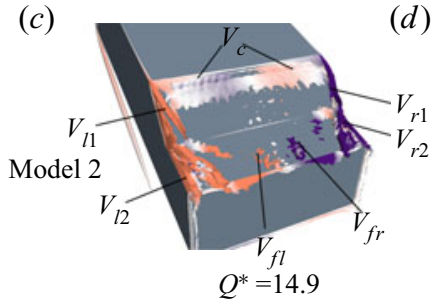

(b)

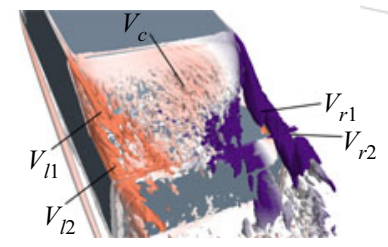

$Q^{*}=4.5$

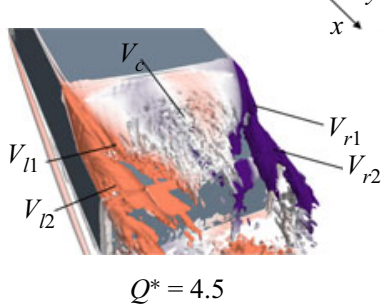

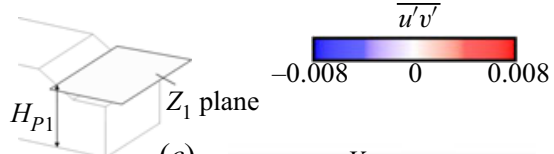

(e)

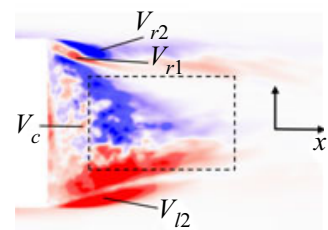

$(f)$

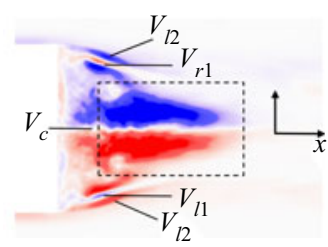

Figure 10. Flow structures visualized by iso-surfaces of mean $Q$-criterion. For $Q^{*}=14.9$ : $(a)$ Model 1, during $S_{b} ;(b)$ Model 2, during $S_{c}$. For $Q^{*}=4.5$ : (c) Model 1, during $S_{b} ;(d)$ Model 2, during $S_{c}$. Distributions of the mean Reynolds shear stress component, $\overline{u^{\prime} v^{\prime}}$, on the $Z_{1}$ plane behind the slant, located at $H_{P 1}=0.844 H$ above the bottom of the model (half-height of the slant): (e) Model 1, during $S_{b} ;(f)$ Model 2, during $S_{c}$.

figure 6(a). Furthermore, as shown in figure $10(b), V_{c}$ deflects to the left-hand side interacting with $V_{l 1}$. On the right-hand side, $V_{r 1}$ and $V_{r 2}$ merging to form $V_{r}$ moving downstream are not affected by $V_{c}$. However, during $S_{c}$, as shown in figure $10(c, d), V_{c}$ separates from both sides of the upper slant. In this case, $V_{c}$ is in the centre. Thus, $V_{l 1}$ or $V_{r 1}$ on either side is not disturbed by $V_{c}$. 


\section{K. He and others}
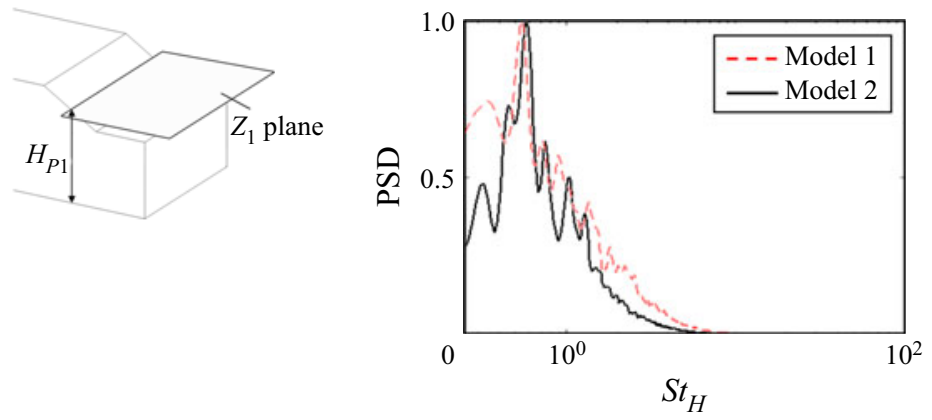

Figure 11. The PSD of pressure signals sampled on the $Z_{1}$ plane behind the slant. The considered sampling period for Model 1 is $S_{b}$ and for Model 2 is $S_{c}$.

The status of $V_{c}$ is found to be relevant to the fragmented structures on the deck. Once $V_{c}$ deflects to the left-hand side, $V_{f r}$ on the right-hand side of the deck is more dominant than $V_{f f}$. Likewise, during the symmetric state, $V_{f l}$ and $V_{f r}$ are equivalent to maintain $V_{c}$ to the centre. The reverse $V_{f l}$ and $V_{f r}$ located on the deck are considered related to the 'decklid' vortices observed by Jenkins (2000), who suggested that these two vortices induce reverse flow over the decklid dominating the separation of a notchback car.

The distribution of $\overline{u^{\prime} v^{\prime}}$ behind the slant given in figure $10(e, f)$ suggests that, during $S_{b}$, both $V_{r 1}$ and $V_{r 2}$ can be observed on the right-hand side. However, on the left-hand side, only $V_{l 2}$ appears due to the interaction of $V_{l 1}$ and $V_{c}$. During $S_{c}$, on the other hand, the C-pillar vortex containing two subvortices can be clearly found on each side. Comparing $\overline{u^{\prime} v^{\prime}}$ inside the dashed frames, one can observe the deflection of $V_{c}$ during $S_{b}$ but the symmetry of $V_{c}$ during $V_{c}$. Moreover, the absolute value of $\overline{u^{\prime} v^{\prime}}$ is found to be higher with the symmetric wake, indicating a more turbulent flow behind Model 2. For this reason, the wake instability involving the unsteadiness of separations requires a deeper analysis.

The FFT algorithm is employed to assess the power spectral density (PSD) function of pressure on the $Z_{1}$ plane behind the slant (see figure 11). The frequency spectrum shows approximated peaks for the two models, giving $S t_{H}=0.53$ for Model 1 and $S t_{H}=0.6$ for Model 2. Therefore, it is interesting to inquire how the flow structures behind the two models show similar primary frequencies but behave inconsistently.

For an in-depth exploration of the unsteady structures in the wake, POD is applied on the pressure field on the $Z_{1}$ plane. For example, figure 12(a) shows the energy content of the first six pressure modes concerning the considered period, the asymmetric state during $S_{b}$. Here, Mode 1 corresponds to the averaged pressure field containing most of the energy. The rest of the modes are distributed in pairs. Figure 12(b) shows the temporary coefficients obtained in the left- and right-hand parts of Mode 2. The two curves shown in red and black are similar but shifted in time, providing the steps to reconstruct the motion of the considered flow structures.

The wake structures recognized by pressure snapshots and the POD distributions with associated modes are presented in figure 13, showing the wake dynamics during $S_{b}$ and $S_{c}$. The flow structures $V_{c}$ associated with the vortex shedding from the shear layer of the slant, and the two C-pillar vortices, $V_{l}$ and $V_{r}$, are distinguished by the instantaneous pressure in figure $13(a, b)$. During $S_{b}, V_{c}$ deflects to the left-hand side to interact with $V_{l}$. Meanwhile, $V_{r}$ extending further downstream on the right-hand side is not affected by $V_{c}$. During $S_{c}$, however, given that $V_{c}$ symmetrically separating from the upper slant is located in the central region, the two $\mathrm{C}$-pillar vortices are equivalent on both sides. 
(a)

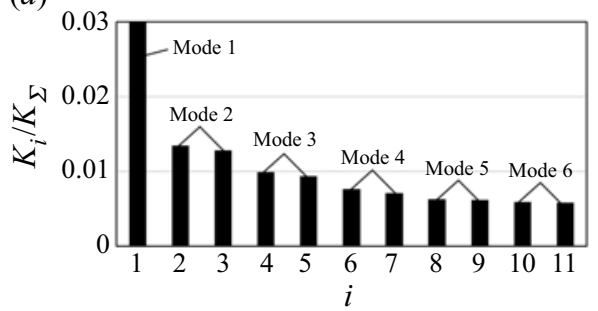

(b)

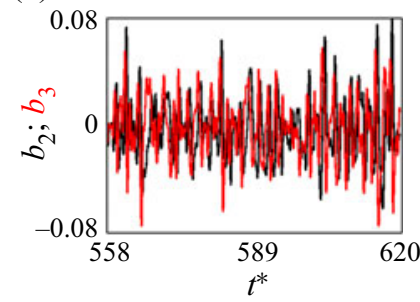

Figure 12. Proper orthogonal decomposition analysis.

(a) Energy content of the first six modes. (b) Temporary coefficients: $b_{2}$ (black) and $b_{3}$ (red).

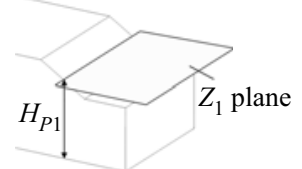

(a) $\begin{gathered}C_{p} \\ \end{gathered}$

(c)

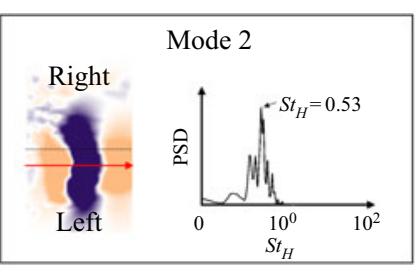

(e)

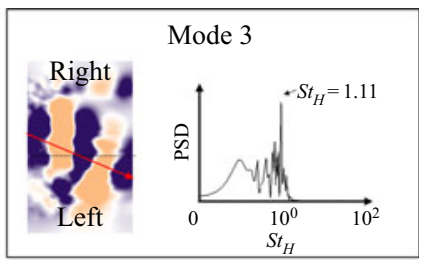

(b)

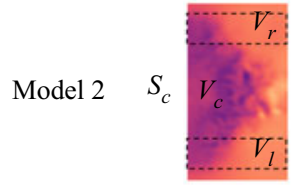

(d)

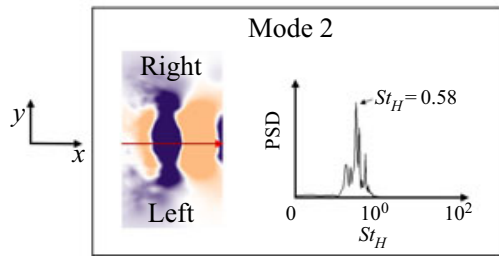

$(f)$

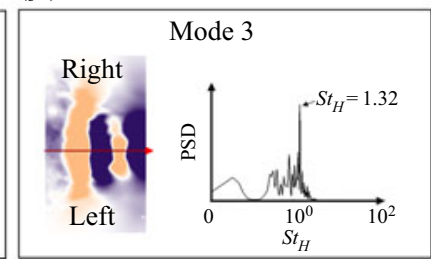

Figure 13. Proper orthogonal decomposition analysis for the pressure projected on the $Z_{1}$ plane. The snapshot of instantaneous pressure: (a) Model 1, during $S_{b}$; $(b)$ Model 2, during $S_{c}$. Spatial distribution and the associated PSD of the temporary coefficient of Mode 2: (c) Model 1, during $S_{b}$; (d) Model 2, during $S_{c}$. Mode 3: (e) Model 1 , during $S_{b} ;(f)$ Model 2, during $S_{c}$. The dotted line is in the central symmetry plane at $y=0$.

The main moving direction of $V_{c}$ is identified in Mode 2 by showing the wave packets, suggesting that $V_{c}$ moves downstream on the left-hand side during $S_{b}$ (figure $13 c$ ), but on the centre during $S_{c}$ (figure 13d). The associated main frequencies of Mode 2 are similar to the results of FFT (figure 11). Therefore, the main frequency containing most of the energy describes the marked separation shedding from the shear layer of the upper slant. Furthermore, expressed by Mode 3, secondary directions of $V_{c}$ are indicated, showing left-hand deflection described by the flow patterns in figure 13(e). On the other hand, in figure $13(f)$, the secondary direction describes $V_{c}$ moving downstream in the centre, following the main direction. The main frequency of Mode 3 is slightly lower for Model 1 than for Model 2. As a result, the wake dynamics shown in figure 13 confirms the wake asymmetry for Model 1 and the symmetry for Model 2.

Focusing on the C-pillar vortices, the dynamics of $V_{l}$ and $V_{r}$ can be identified by POD modes employed on the divided regions shown in figure $13 a, b$. The modality of the 


\section{K. He and others}
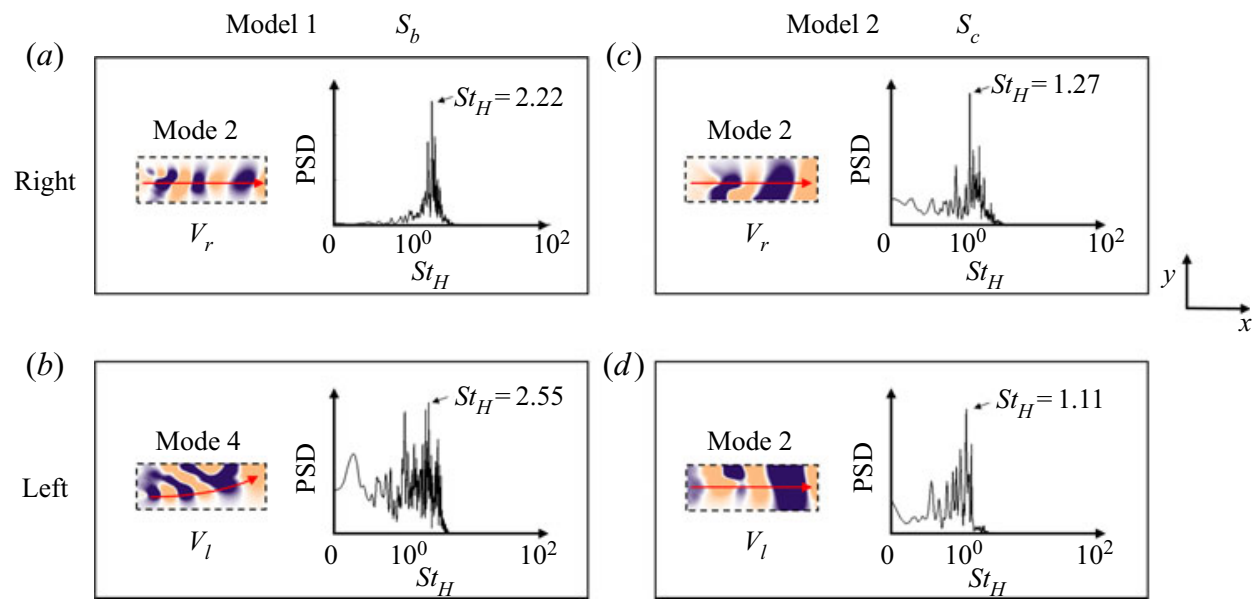

Figure 14. Dynamics of the C-pillar vortex and the associated PSD of the temporary coefficient: $(a) V_{r}$ for Model 1 during $S_{b} ;(b) V_{l}$ for Model 1 during $S_{b} ;(c) V_{r}$ for Model 2 during $S_{c} ;(d) V_{l}$ for Model 2 during $S_{c}$.

C-pillar vortex is presented in figure 14. During $S_{b}$, since $V_{c}$ deflects to the left-hand side, $V_{r}$ moving downstream on the right-hand side can be explicitly captured by Mode 2 with a highly concentrated main frequency of $S t_{H}=2.22$ (see figure $14 a$ ). On the left-hand side, in figure $14(b), V_{l}$ is disturbed by the deflection of $V_{c}$. Therefore, the direction of $V_{l}$, moving downstream with a deviation to the centre, is vague until Mode 4. In the spectrum of the associated frequency, in addition to the main frequency of $V_{l}$ at $S t_{H}=2.55$, peak clusters with lower frequencies are detected due to the interaction with $V_{c}$. However, in agreement with $V_{c}$ moving downstream without deflections, dynamics of $V_{r}$ and $V_{l}$ can be both described by Mode 2 in the symmetric case during $S_{c}$ (see figure $14 c, d$ ). The main frequencies of $V_{r}$ and $V_{l}$ are identified by evident peak values, confirming that the two $\mathrm{C}$-pillar vortices are not affected by $V_{c}$. It can be noted that the frequency of the C-pillar vortex is lower for Model 2 than Model 1, which is attributed to the discrepancy between the two effective backlight angles.

The different behaviour of the wake structures between the asymmetric and symmetric flows influences the aerodynamic drag. In order to discuss the asymmetry of the force signals, the two models are divided into left and right parts. Based on the projected area of the entire frontal model surface, the coefficients of the aerodynamic drag are displayed in figure 15. During $S_{b}$, as shown in figure 15(a), the time history curves and the probability density illustrate that the drag $C_{d}$ is markedly lower on the left part of Model 1, demonstrating an explicit flow asymmetry. In figure $15(b)$, however, $C_{d}$ shows high degrees of consistency between the two parts. Notably, during $S_{c}, C_{d}$ on the two sides is characterized by frequent switches between two highly consistent curves, showing an instability of the symmetric wake.

Considered as a whole, the averaged drag of the entire Model 1 is $\overline{C_{d}}=0.324$, being lower than $\overline{C_{d}}=0.345$ of Model 2, which is related to the base pressure suggested by Roshko (1993). The averaged base pressure $\overline{C_{p B i}}$ on the rear of Model 1 and Model 2 is presented in figure 16. The difference of $\overline{C_{p B i}}$ between the two models can be found on the slant and the deck, showing lower absolute values obtained for Model 1. By the base pressure definition given in $\S 2.3$, the lower absolute value of $\overline{C_{p B i}}$ suggests a lower value of negative pressure on the rear surface, leading to a lower drag of the model. Therefore, $\overline{C_{p B i}}$ shown in figure 16 follows the lower $\overline{C_{d}}$ observed for Model 1 . Moreover, it can be 

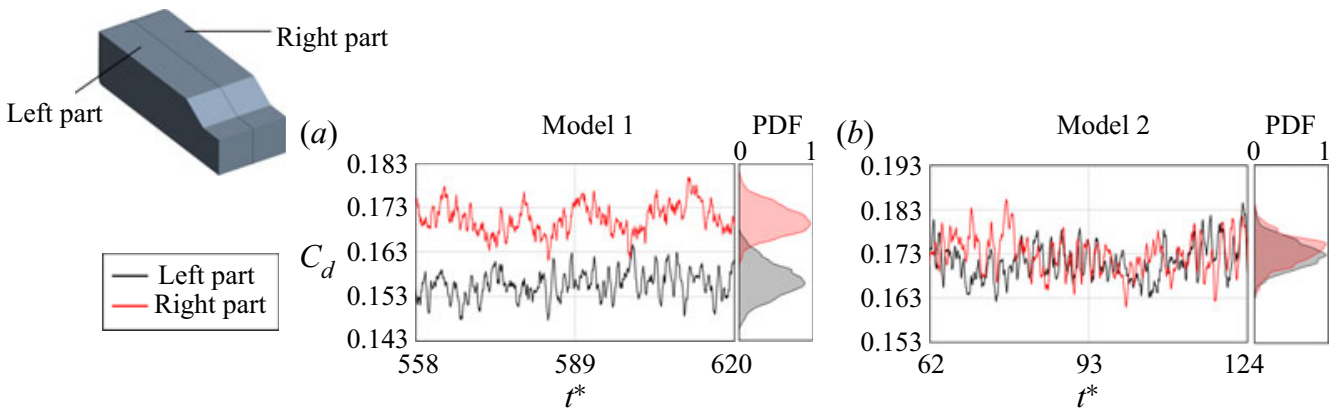

Figure 15. Time history curves of the aerodynamic drag coefficient $C_{d}$ : (a) Model 1, during $S_{b}$; Model 2 , during $S_{c}$. The right-hand side of each graph shows PDF of $C_{d}$.

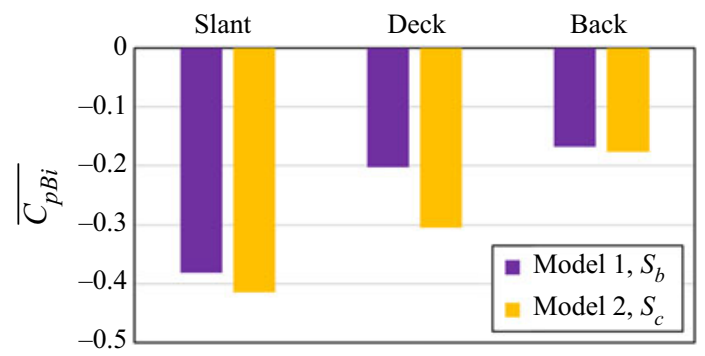

Figure 16. Averaged base pressure $\overline{C_{p B i}}$ on the rear model; $i \in\{s, d, b\}$ stands for the slant, the deck and the back wall.

seen from figure 6 that, compared to Model 1 (figure $6 a$ ), the flow structure $V_{c}$ has a larger contact area attached to the slant and the deck of Model 2 because $V_{c}$ forms on both sides (figure $6 b$ ). For this reason, the negative pressure inside $V_{c}$ contributes to the higher absolute value of the negative base pressure for Model 2.

\subsection{Switching process of the wake bi-stability}

For the wake bi-stability of squareback Ahmed bodies, the asymmetric wake has been observed to randomly switch to the opposite mirrored state (Grandemange et al. 2013a,b; Östh et al. 2014; Volpe et al. 2015; Dalla Longa et al. 2019; Haffner et al. 2020). In terms of the notchback configuration, the switching process of wake bi-stability was experimentally observed by Cogotti (1986) and Lawson et al. (2007). In this work, the first numerical result showing a switching period for the bi-stability behind the notchback body (Model 1) is presented, denoted $S_{d}$ during $t^{*} \in[63,125]$, shown figure 17( $a$ ). The switch of the wake is identified by $\partial C_{p s} / \partial y$ dropping from positive to negative values during $S_{d}$, showing bi-stability. The expectation for the second wake switch has not been achieved during the simulation lasting for 30 flow-through passages through the domain on account of the large time scale of the bi-modality. However, the possibility of triggering the switching process is signified by the illustration of $\partial C_{p s} / \partial y$ repeatedly approaching 0 . Therefore, the time period, $S_{e}$ during $t^{*} \in[252,314]$, showing the attempt to switch, is selected to compare with $S_{d}$ to analysis the switching process.

Probability distributions of base pressure gradients on the rear of Model 1, during $S_{d}$ and $S_{e}$, are presented in figure 18. In figure 18(a), concentrations with both positive and negative values are observed on the rear body, particularly on the slant and 


\section{K. He and others}
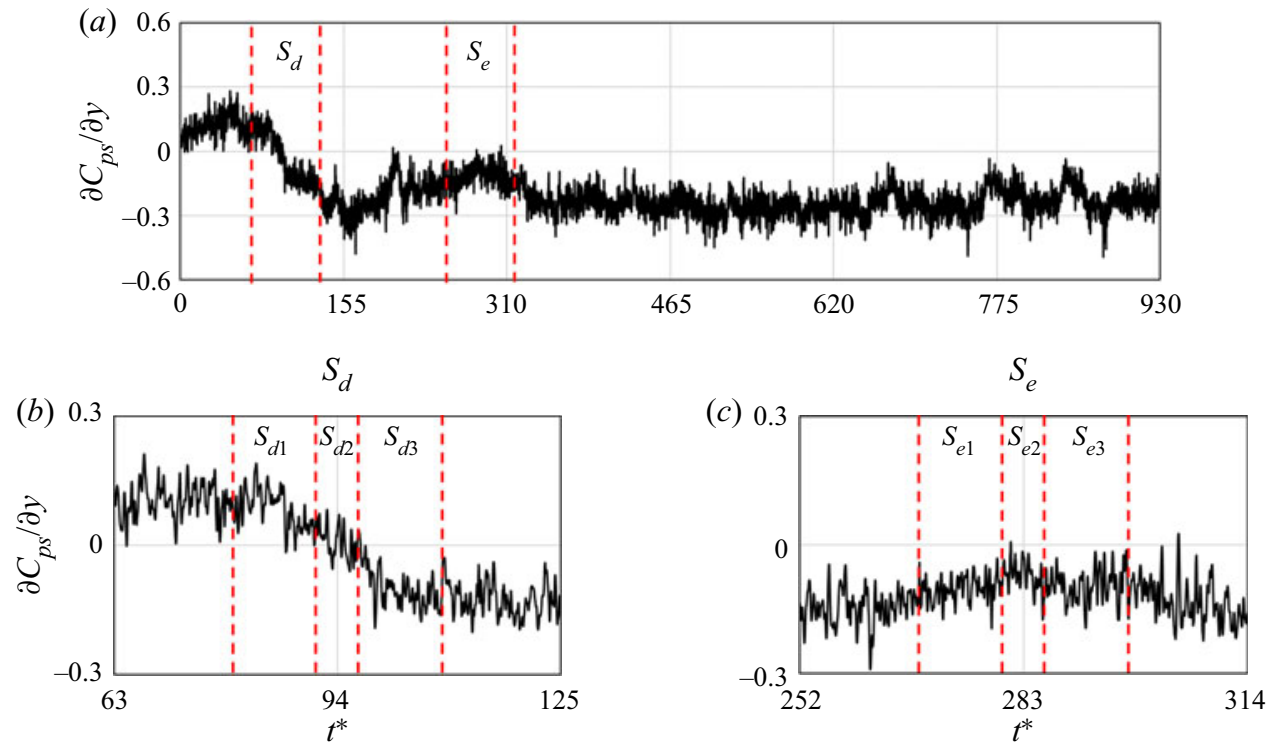

Figure 17. Time history curves of base pressure gradients on the slant surface: $(a)$ the entire simulation for Model 1; (b) the switching period during $S_{d}$, in which $S_{d 1}$ is $t^{*} \in[84,92], S_{d 2}$ is $t^{*} \in[92,96], S_{d 3}$ is $t^{*} \in$ [96, 104]; $(c)$ the attempt to switch during $S_{e}$, in which $S_{e 1}$ is $t^{*} \in[273,281], S_{e 2}$ is $t^{*} \in[281,285], S_{d 3}$ is $t^{*} \in[285,293]$.

the deck. Therefore, the identification of the wake bi-stability behind Model 1 is indicated, suggesting that the wake during $S_{d}$ experiences two asymmetric mirrored states. However, in figure $18(b)$, it is evident that the pressure gradients on each rear surface are concentrated on one side, showing the absence of the opposite state.

To focus on the switching periods, the central parts of $S_{d}$ and $S_{e}$ are extracted, denoted $S_{d 1-3}$ and $S_{e 1-3}$, respectively, shown in figure $17(b, c)$. During these periods, the wake starts to switch $\left(S_{d 1}\right.$ and $\left.S_{e 1}\right)$, is switching $\left(S_{d 2}\right.$ and $\left.S_{e 2}\right)$, and reverses to the opposite state $\left(S_{d 3}\right)$ or returns to the original state $\left(S_{d 3}\right)$.

The mean streamlines and the spanwise vorticity distribution during $S_{d 1-3}$ and $S_{e 1-3}$ are presented in figure 19. For the switching period $S_{d 1-3}$, as indicated in figure $19(a)$, the flow separating from the upper part of the slant reattaches to the deck on both sides. Before the switch, $S_{d 1}$, the reattachment length $L_{R}$ on the left-hand side of the deck is found to be longer than that on the right-hand side due to the larger separation angle $\theta_{1}$. As a consequence, the downstream separation angle $\theta_{2}$ has a smaller value on the left-hand side. During $S_{d 2}$, once the wake is switching, a temporary symmetric state with comparable separations and reattachments on the two sides can be observed. After a successful switch, the wake turns asymmetric on the opposite side during $S_{d 3}$. Angle $\theta_{1}$ on the left-hand side is found to be larger, resulting in a longer $L_{R}$ and a smaller $\theta_{2}$.

Figure $19(b)$ shows the separations and the reattachments during the period $S_{e 1-3}$. The wake asymmetry during $S_{e 1}$ is characterized by a smaller $\theta_{1}$, shorter $L_{R}$ and larger $\theta_{2}$ on the left-hand side. During $S_{e 2}$, although the mean flow fails to show a perfect symmetry, the asymmetry is found to become weaker than for $S_{e 1}$. During $S_{e 3}$, the smaller $\theta_{1}$, shorter $L_{R}$ and larger $\theta_{2}$ are still found on the left-hand side, showing that the wake returns to the original asymmetric state as a result of the failed switch.

Flow mechanisms of the wake switch are analysed from the perspective of the flow structures behind the slant. The instantaneous distribution of the streamwise vorticity 

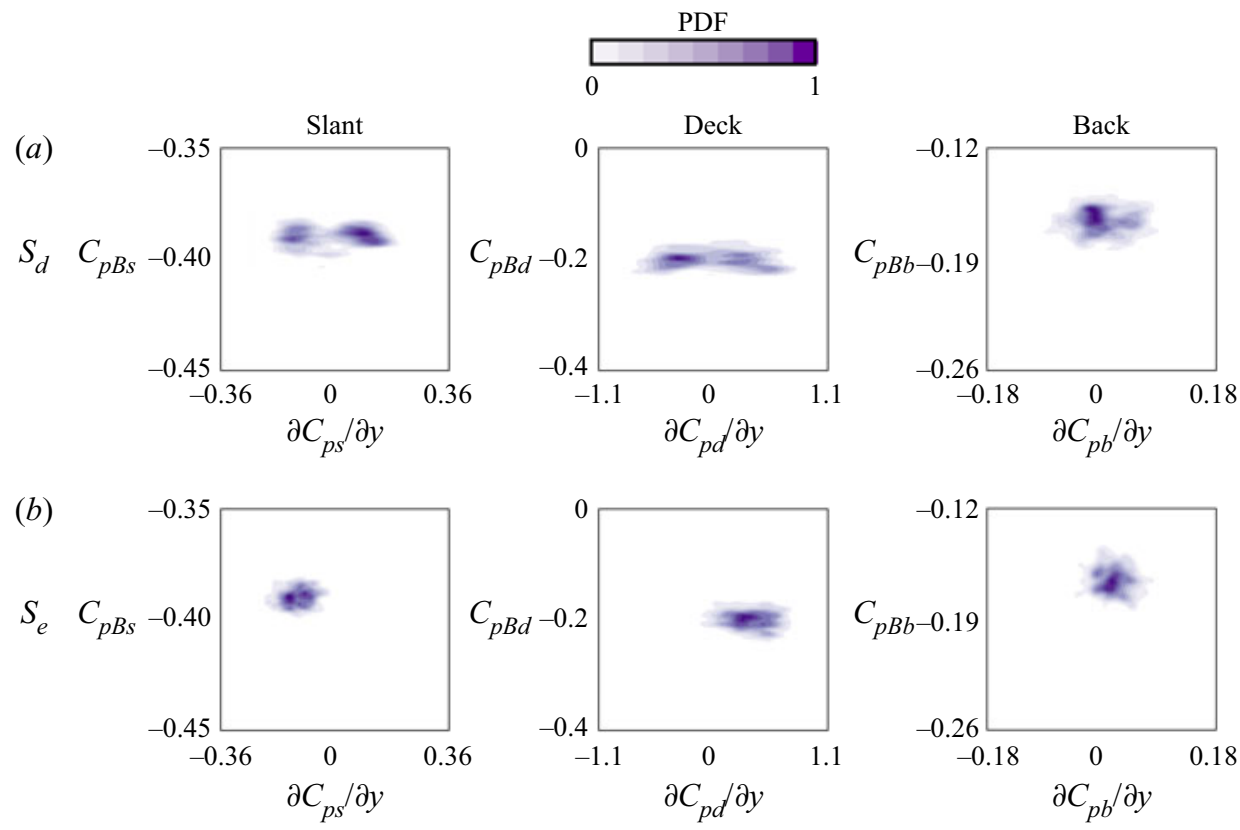

Figure 18. From left to right, PDFs of the base pressure gradients against the base pressure on the slant, the deck and the back wall: $(a)$ during $S_{d}$, the successful switching period; $(b)$ during $S_{e}$, the failed switching period.

showing separations behind the roof's trailing edge is presented in figure 20. It can be seen that the wake structure $V_{c}$ consists of two substructures, $V_{c l}$ and $V_{c r}$, forming on the left- and right-hand sides of the upper slant, respectively. On the left-hand side, $V_{c l}$ moves downstream, deflecting to the right-hand side. On the right-hand side, $V_{c r}$ deviates towards the left-hand side. Using the POD method, modal analysis is applied for the two switching periods $S_{d 1-3}$ and $S_{e 1-3}$, with the aim of exploring the dynamics of the wake structures, as shown in figure 21 .

Snapshots of the wake structures behind the upper part of the slant during the period $S_{d 1-3}$ in which a successful switch of the bi-stability occurs are presented in figure $20(a)$. At $t^{*}=84, V_{c l}$ is dominant, showing a larger separation region attached to the upper slant. Meanwhile, $V_{c}$ combining the downstream parts of $V_{c l}$ and $V_{c r}$ is located at the right-hand side. In figure $21(a)$, the main direction of $V_{c}$, moving downstream on the right-hand side, is described by Mode 2 of POD, showing the wake dynamics before the switch. Moving to $t^{*}=89$, the region of $V_{c r}$ gradually becomes larger. This can be explained by an energetic movement of $V_{c r}$ in the secondary direction, deflecting to the left, as captured by Mode 3 in figure 21 $(a)$. However, during $S_{d 1}$ the movement of $V_{c l}$ is invisible in the first three POD modes. At $t^{*}=94$, the structures $V_{c l}$ and $V_{c r}$ are found to be of similar extension, resulting in a temporary symmetric state. In figure $21(b)$, the direction of the flow structures showing the movement in the downstream direction is presented with Mode 2 and Mode 3. After the successful switch, the extension of $V_{c r}$ continues to increase, as shown in the snapshots at $t^{*}=99$ and $t^{*}=104$. Therefore, $V_{c}$ starts to move downstream at the left-hand side, as described by the distribution of the streamwise vorticity and by Mode 2 given in figure 21(c). This means that the asymmetric wake reverses to the opposite state after the switch. Moreover, it is noted that Mode 3 in figure 21(c) indicates a secondary direction 


\section{K. He and others}

(a)

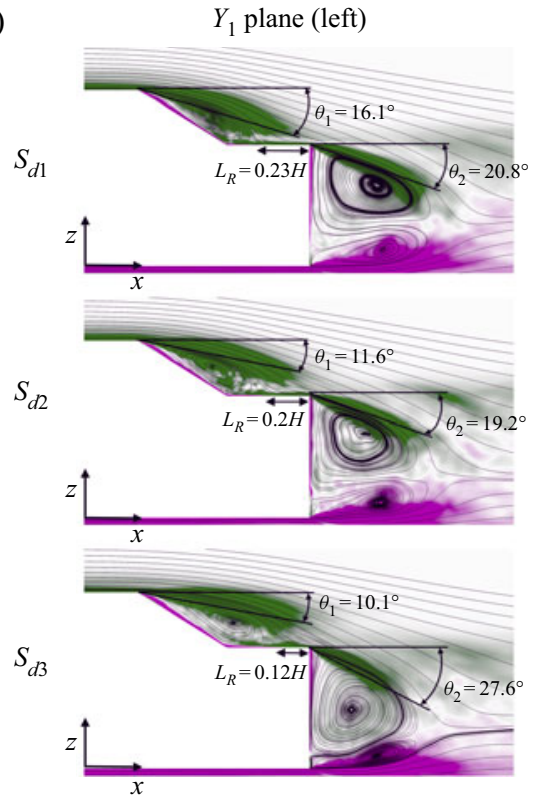

(b)

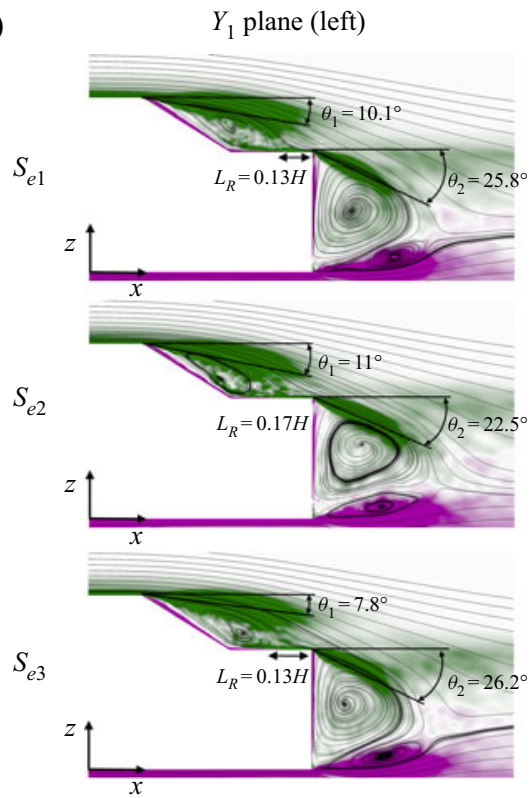

$Y_{2}$ plane (right)

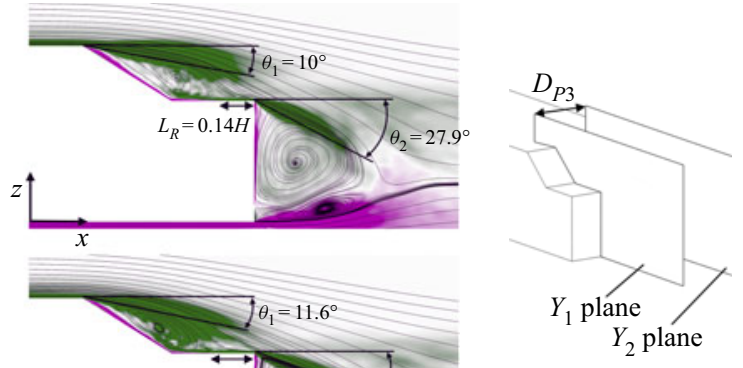

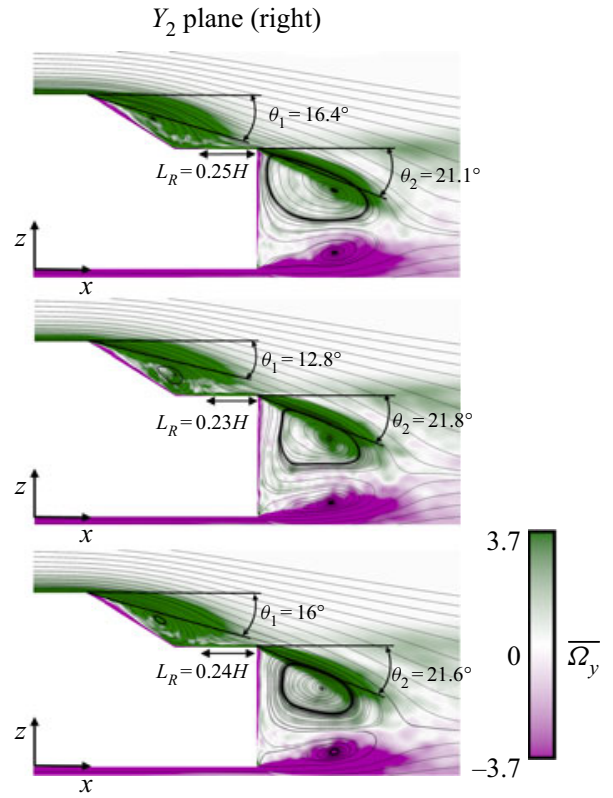

Figure 19. Mean streamlines and spanwise vorticity, $\overline{\Omega_{y}}$, on the left $Y_{1}$ plane (left-hand column) and the right $Y_{2}$ plane (right-hand column), the distance between $Y_{1}$ and $Y_{2}$ planes being $D_{P 3}=0.5 \mathrm{~W}$ : $(a)$ during $S_{d 1-3}$; (b) during $S_{e 1-3}$.

showing the movement of $V_{c r}$ with a deflection to the left. This means that $V_{c r}$ remains more energetic than $V_{c l}$, showing the stability of the asymmetric wake after the switch.

Snapshots of the wake structures during the period of the attempt to switch, $S_{e 1-3}$, for the observation of the process of the failed switch, are presented in figure 20(b). At $t^{*}=273$, the separation region of $V_{c r}$ is larger than that of $V_{c l}$. At the same time, $V_{c}$ is 
(a)

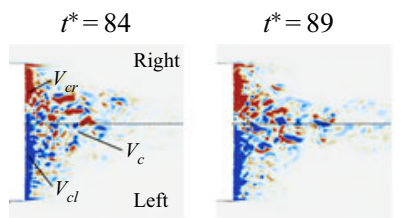

$t^{*}=94$

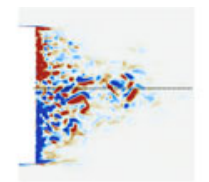

(b)

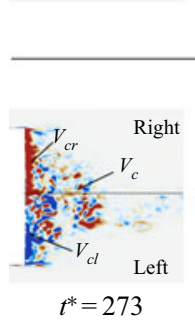

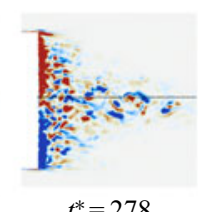

$t^{*}=278$
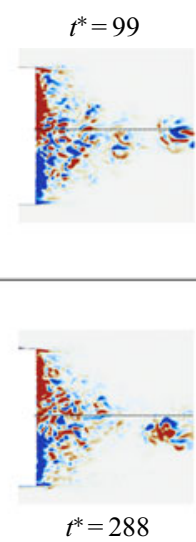

$t^{*}=104$
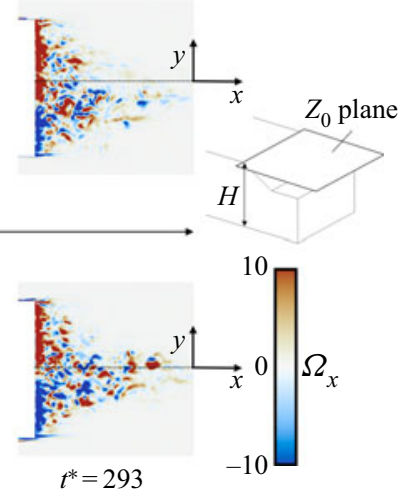

Figure 20. Instantaneous distribution of the streamwise vorticity, $\Omega_{x}$, on the $Z_{0}$ plane behind the roof's trailing edge: (a) during $S_{d 1-3}$; (b) during $S_{e 1-3}$. The dotted line is the central symmetry plane at $y=0$.

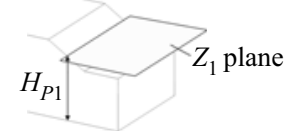

(a)

(b)

$S_{d 2}$

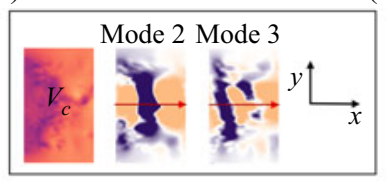

(c)

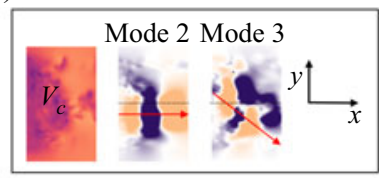

(e)

(f) $\quad S_{e 3}$

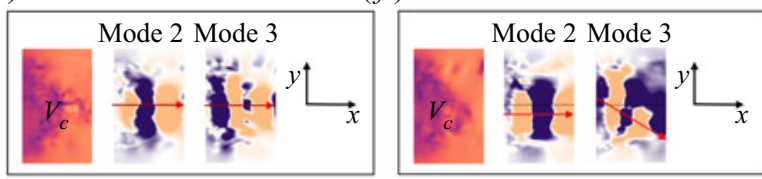

Figure 21. Proper orthogonal decomposition analysis for the pressure distribution projected on the $Z_{1}$ plane behind the slant during the switching process of the wake bi-stability. Each panel shows a snapshot of instantaneous pressure, Mode 2 and Mode 3. During the temporary states: (a) $S_{d 1}$; (b) $S_{e 1}$; (c) $S_{d 2}$; (d) $S_{e 2}$; (e) $S_{d 3} ;(f) S_{e 3}$. The dotted line is in the central symmetry plane at $y=0$.

mainly on the left-hand side, moving downstream as described by Mode 2 in figure $21(d)$. The asymmetric wake state before the attempt to switch is unstable, due to deflection to the right, as seen in Mode 3 in figure 21 $(d)$. This proves that the movement of $V_{c l}$ is more energetic, despite a smaller extension. Thereby, the region of $V_{c l}$ becomes larger at $t^{*}=278$ and matches $V_{c r}$ at $t^{*}=283$, showing the transition of the wake to the temporary symmetric state. The movement of $V_{c}$ to the downstream wake in the centre is described by Mode 2 and Mode 3 in figure 21(e). Although the temporary symmetric state at $t^{*}=283$ is found to be similar to that at $t^{*}=94$, the wake fails to switch as the region of $V_{c r}$ remains larger and $V_{c}$ stays on the left-hand side (see snapshots at $t^{*}=288$ and $t^{*}=293$ ). This means that the wake returns to the original asymmetric state after the failed switch. In figure $21(f)$, the direction of $V_{c}$ moving downstream on the left-hand side is indicated in 


\section{K. He and others}

(a)

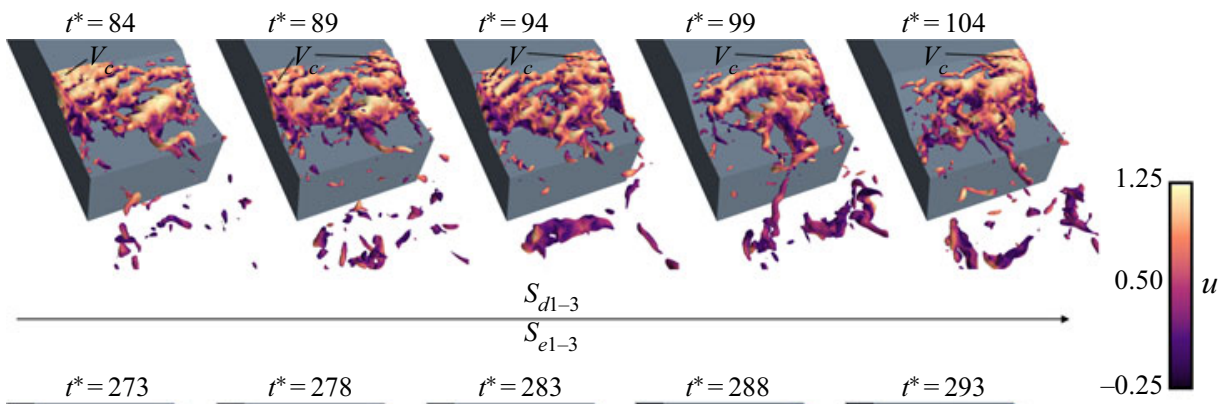

(b)

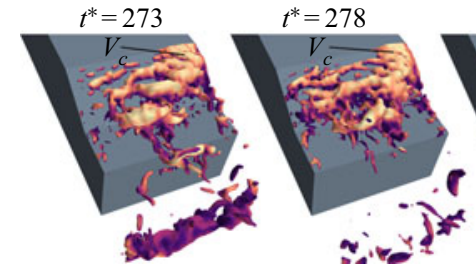

$t^{*}=288$

$t^{*}=293$

$-0.25$
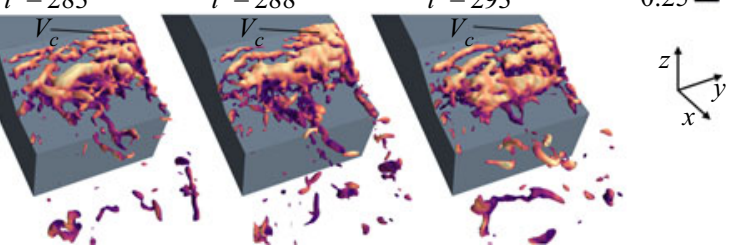

Figure 22. Instantaneous iso-surfaces of pressure at $C_{p}=-0.8$, coloured by the instantaneous streamwise velocity, $u$ : (a) during $S_{d 1-3}$; (b) during $S_{e 1-3}$.

Mode 2. Furthermore, Mode 3 in figure 21 $(f)$ indicates the deflection to the left, showing that $V_{c r}$ with a larger region tends to be more energetic. This produces a stable wake asymmetry after the failed switch.

In order to present the three-dimensional flow structures behind the slant during $S_{d 1-3}$ and $S_{e 1-3}, V_{c}$ identified by evaluating iso-surfaces of instantaneous pressure at $C_{p}=-0.8$ is displayed in figure 22. Particularly, figure 22(a) shows the process of the successful switch. In the beginning, at $t^{*}=84, V_{c}$ forms at the upper-left of the slant with a deflection to the right-hand side. Moving to $t^{*}=89$, with the deflection to the right, $V_{c}$ starts to form at the upper-right corner of the slant. Afterwards, $V_{c}$ moves to the right-hand side, showing the appearance of $V_{c}$ on the two upper corners of the slant, as described in the snapshot at $t^{*}=94$. As indicated in snapshots at $t^{*}=99$ and $t^{*}=104, V_{c}$ disappears on the upper-left corner and eventually switches to the right-hand side, completing the switching process. As for the process of the failed switch, figure 22(b) shows that, at $t^{*}=$ $273, V_{c}$ forms at the upper-right corner of the slant with a deflection to the left. However, in the following procedure, although showing an attempt to reach the switch, the left shift of $V_{c}$ is insufficient to force the structure to form at the left-hand side of the slant, culminating in the failed switch.

Observations of figures 20-22 allow the deduction that, as described by the secondary direction, the deflection of the near-wall structures behind the slant to the opposite side triggers the process of the switch. The decisive factor activating a successful switch is the consequence of the deflection that can drive the wake structures to the opposite side after a temporary symmetric state. Once the flow structure, $V_{c}$, starts to form at the upper slant on the opposite side, a switch of the bi-stability is finalized. On the contrary, when the deflection of $V_{c}$ is inadequate to sustain its formation on the opposite side, the wake fails to switch and returns to the initial state. As a result, the wake develops into a stable asymmetric state after the successful or failed switching process.

Furthermore, the downstream toroidal vortex is presented in figure 23. The toroidal vortex behind the squareback Ahmed body observed by Dalla Longa et al. (2019) was sensitive to the switching process of the wake bi-stability. For the notchback Ahmed body, it is found that the status of the toroidal vortex is dependent on the wake state. 
(a)

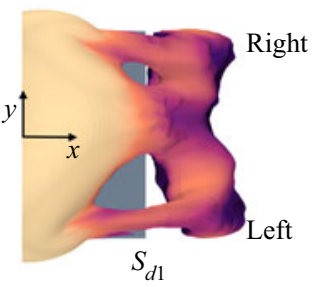

(b)

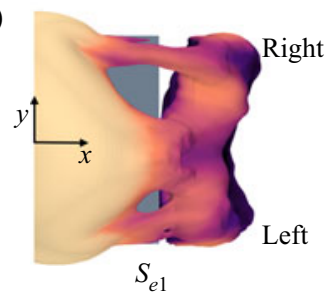

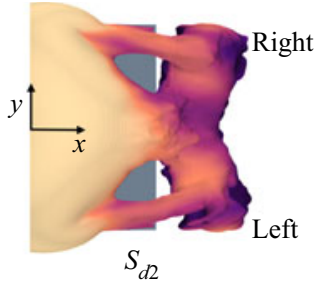

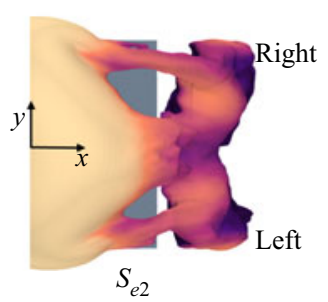

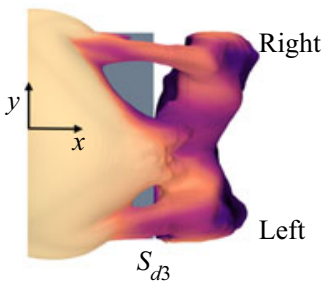
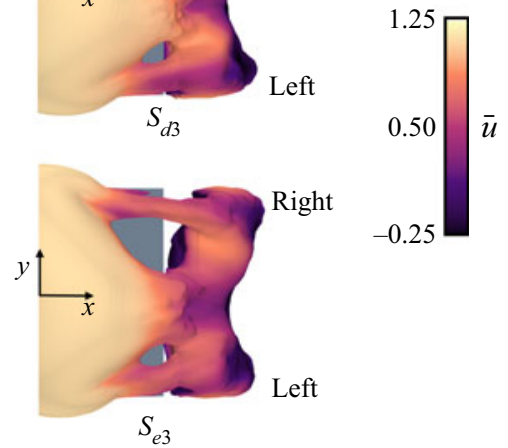

Figure 23. Iso-surfaces of mean pressure at $\overline{C_{p}}=-0.4$, coloured by the mean streamwise velocity, $\bar{u}$ : (a) during $S_{d 1-3} ;(b)$ during $S_{e 1-3}$.

In figure 23(a), during $S_{d 1}$ before the switch, the left part of the toroidal vortex tilts backwards. Then, the toroidal vortex tilts on both sides when the wake is switching during $S_{d 2}$, in which a temporary symmetry is observed. After the successful switch, following the wake turning to be asymmetric on the opposite side, the right-hand part of the toroidal vortex tilts backwards. For the period of the attempt to switch shown in figure 23(b), the right-hand part of the toroidal vortex tilts backwards during $S_{e 1}$ before the switch. The temporary symmetry of the toroidal vortex is thereby formed as both sides tilt during $S_{e 2}$. However, the difference is that the right-hand part of the toroidal vortex tilts again after the failed switch, as observed during $S_{e 3}$. From figure 23, the underlying mechanism of the downstream flow structure is found sensitive to the upstream wake state in the notchback region.

\section{Conclusions}

The LES investigation for flows around two notchback Ahmed bodies with different effective backlight angles, $17.8^{\circ}$ for Model 1 and $21.0^{\circ}$ for Model 2, shows the wake asymmetry and symmetry following the experimental results observed by Sims-Williams et al. (2011).

A random switch of two stable asymmetric mirrored states is observed during the simulation for Model 1, showing the bi-stability. The wake behind Model 2 results in a stable symmetric state. The wake states are identified by the horizontal base pressure gradients. The asymmetry of the flow is attributed to the asymmetric separation angles behind the upper slant, leading to distinct reattachment length on two sides of the deck. The form of wake structures behind the slant and the downstream back wall follows the asymmetry. The modal analysis applying POD confirms the asymmetric wake dynamics for Model 1 and the symmetry for Model 2. The main direction moving downstream on one side and the secondary direction indicating the deflection are described for wake structures behind the slant of Model 1. However, these main and secondary directions for Model 2 suggest that the wave packets move downstream in the centre with no deflections. The wake frequency involving the separation from the upper slant and the two C-pillar vortices are presented. The wake asymmetry resulting in the aerodynamic drag difference between the left- and right-hand parts of the model is observed. 


\section{K. He and others}

The random switch of the bi-stable wake is for the first time observed for a notchback vehicle in numerical simulations. A switch and one of the attempts to switch are observed for Model 1. The successful and failed switches are identified using the base pressure gradients and wake structures. The secondary direction of the near-wall vortices behind the slant deflecting the wake structures to counteract the asymmetry drives the wake to become temporarily symmetric, triggering the switching process of the wake bi-stability. The successful switch is attributed to the deflection that consequently forces the flow structure to form on the opposite side of the slant. After a successful or failed switching process, the wake develops into the stable asymmetric state.

The present work helps to explore the mechanism of the wake bi-stability behind a notchback vehicle, promoting an understanding of bluff body flows. The results suggest that the bi-stable wake state is associated with the development of flow structures behind the slant.

Acknowledgements. Computations were performed at SNIC (Swedish National Infrastructure for Computing) at the National Supercomputer Center (NSC) at LiU. The authors are thankful to the three anonymous reviewers for their helpful comments.

Funding. The authors acknowledge financial support from the China National Key R\&D Program during the 13th Five-year Plan Period, grant no. 2020YFA0710900. K.H. acknowledges financial support from the China Scholarship Council, grant no. 201906370096, and the Postgraduate Research and Innovation Project of Central South University, grant no. 1053320183303.

Declaration of interests. The authors report no conflict of interest.

Author ORCIDs.

(1) Kan He http://orcid.org/0000-0003-2798-7338;

(1) Guglielmo Minelli http://orcid.org/0000-0002-0069-8168;

D Siniša Krajnović http://orcid.org/0000-0001-8421-9883.

\section{Appendix A. Grid independence examination}

Multi-block hexahedral conforming computational grids are made using the pointwise grids generator. Numerical accuracy is established by performing simulations based on the fine, the medium and the coarse meshes for Model 1, containing $4.2 \times 10^{7}, 3.2 \times 10^{7}$ and $2.2 \times 10^{7}$ cells, respectively. Meshes are generated by mirroring from one side to the other side, ensuring perfect mesh symmetry and avoiding unexpected effects on the asymmetry of the flow. A slice of the medium mesh is shown in figure 24. The computational cells are denser close to the surface of the model, and the spatial resolution around the slant and the deck is much finer than that around the rest of the model. The reason for this is that the separation of the flow and the vortices that form asymmetric or symmetric flows in this region need to be accurately calculated. The first layer of the computational cells throughout the surface of the model has a height of $3 \times 10^{-4} \mathrm{H}$. The wall-normal growth factor near the model is 1.05 , allowing 70 grid layers attached to the surface to resolve the shear-layer flow. Besides, to avoid the influence of the clearance between the bottom of the body and the ground, the computational cells near the ground are concentrated. The height of the first layer near the ground is $4 \times 10^{-4} \mathrm{H}$ in the $z$ direction with a growth factor of 1.2 .

The spatial resolutions of the three meshes are listed in table 1. All grids have the same resolution in the wall-normal direction, giving $n^{+}<1$. However, the grids are stretched in the streamwise and spanwise directions for the medium and the coarse meshes. For each mesh, the spatial resolution of the flow around the middle body has the same $n^{+}$ and $\Delta_{l}^{+}$as the rear part, but the streamwise resolution is more relaxed. The reason is that 


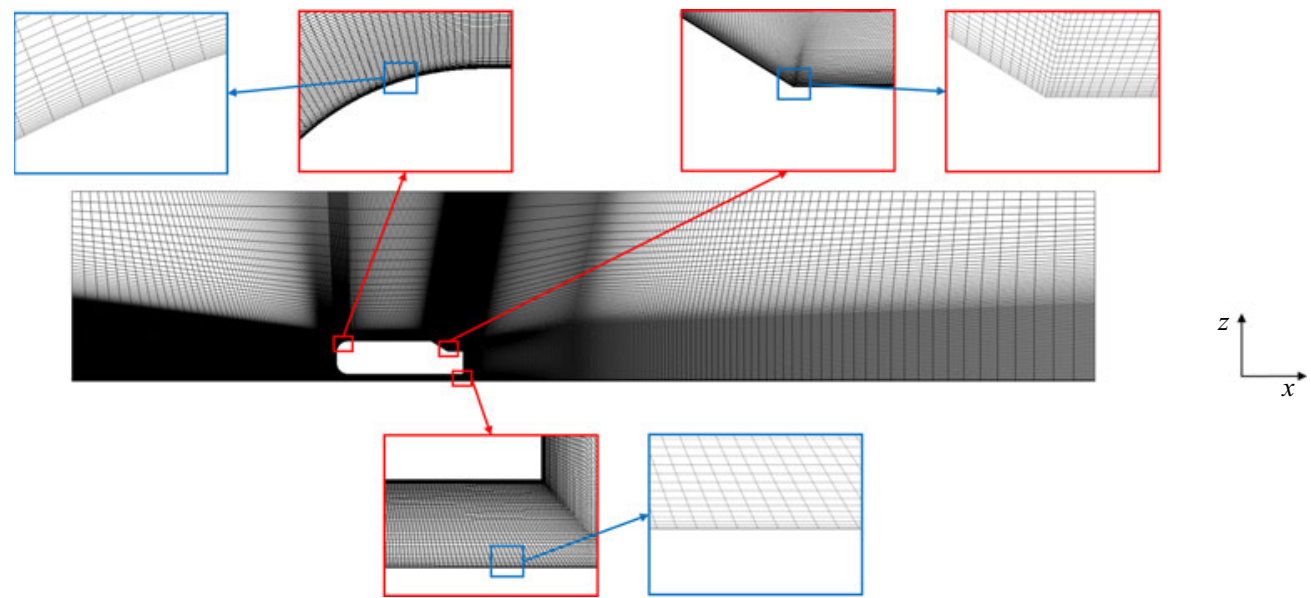

Figure 24. Side view of the mesh on the central plane located at $y=0$.

\begin{tabular}{lccccc}
\hline & & Wall-normal & \multicolumn{2}{c}{ Streamwise resolution } & Spanwise \\
resolution & Middle part & reser of cells \\
Fine mesh & $4.2 \times 10^{7}$ & $n^{+}<1$ & $3<\Delta_{s}^{+}<25$ & $3<\Delta_{s}^{+}<40$ & $3<\Delta_{l}^{+}<20$ \\
Medium mesh & $3.2 \times 10^{7}$ & $n^{+}<1$ & $3<\Delta_{s}^{+}<30$ & $3<\Delta_{s}^{+}<75$ & $3<\Delta_{l}^{+}<25$ \\
Coarse mesh & $2.2 \times 10^{7}$ & $n^{+}<1$ & $3<\Delta_{s}^{+}<40$ & $3<\Delta_{s}^{+}<100$ & $3<\Delta_{l}^{+}<30$
\end{tabular}

Table 1. Details of the grids.

(a)

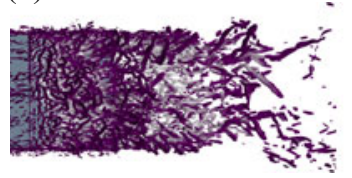

(b)

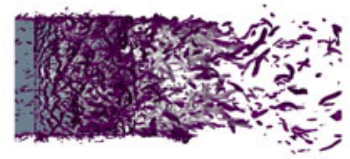

(c)

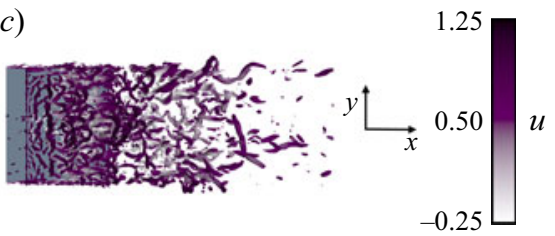

Figure 25. Flow structures behind the slant, visualized by the instantaneous iso-surface of the $Q$-criterion at $Q=2.5 \times 10^{6}:(a)$ fine mesh; $(b)$ medium mesh; (c) coarse mesh.

around the middle body, grids are stretched for saving computing resources since the local surrounding flow is undisturbed.

Qualitative visualization of the second invariant of the velocity gradient, $Q$-criterion, obtained by the fine, the medium and the coarse meshes, is given in figure 25 . This picture shows the resolution level of very large turbulent structures in the wakes. A higher resolution is provided with finer grids, allowing superior turbulent structures to be resolved. From this visualization, a good agreement between the medium and the fine meshes is observed, showing that the medium mesh can result in precise flow structures in this flow.

Since the separations around the slanted rear have proved to be the most challenging in flow predictions for the hatchback Ahmed body (Serre et al. 2013), wakes behind the slant of the notchback geometry are examined in figure 26 . The mean vertical vorticity and streamlines projected on the $Z_{1}$ plane are presented in figure $26(a-c)$. The expected wake 


\section{K. He and others}

(a)

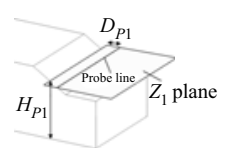

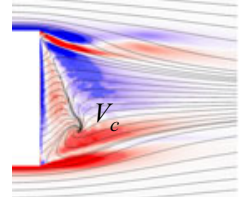

(b)

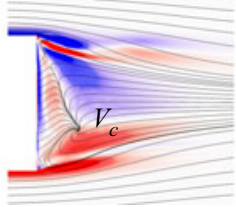

(c)

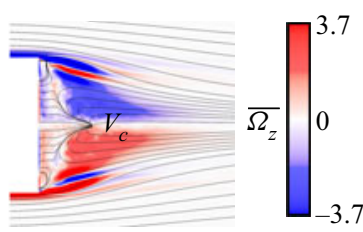

(d)

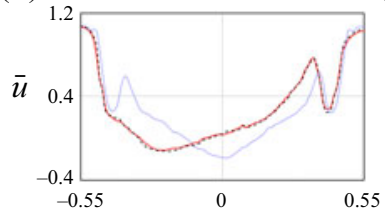

$(g)$

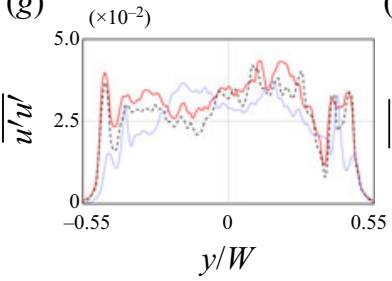

(e)

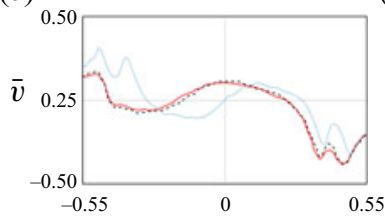

$(f)$

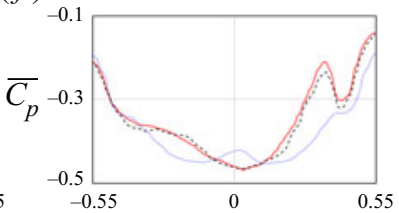

(i)

(h) $\left(\times 10^{-2}\right)$

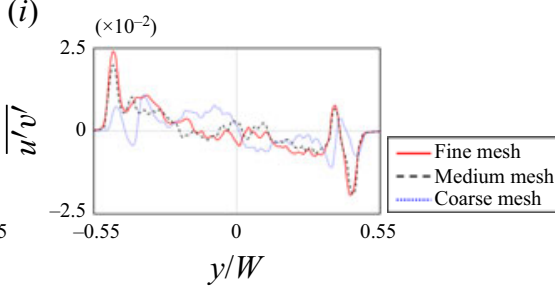

Figure 26. Mean streamlines and the distribution of vertical vorticity, $\overline{\Omega_{z}}$, projected on the $Z_{1}$ plane: $(a)$ fine mesh; (b) medium mesh; (c) coarse mesh. Profiles on the probe line, located at $D_{P 1}=0.3 H$ away from the slant: $(d)$ mean streamwise velocity, $\bar{u}$; $(e)$ mean spanwise velocity, $\bar{v} ;(f)$ mean pressure, $\overline{C_{p}} ;(g)$ mean Reynolds streamwise stress component, $\overline{u^{\prime} u^{\prime}}$; $(h)$ mean Reynolds spanwise stress component, $\overline{v^{\prime} v^{\prime}}$; (i) mean Reynolds shear stress component, $\overline{u^{\prime} v^{\prime}}$.
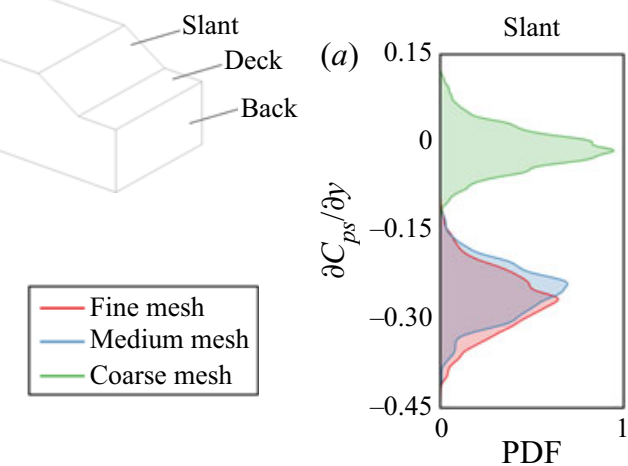
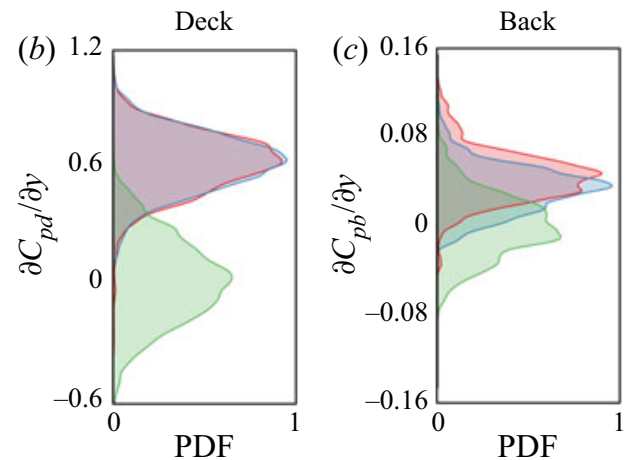

Figure 27. The PDF of base pressure gradients on the surfaces of the rear model: (a) slant; (b) deck; $(c)$ back.

asymmetry is obtained with the fine and the medium meshes, indicating the deflection of the flow structure $V_{c}$ to the left-hand side. However, the wake resulting from the coarse mesh presents an unexpected symmetric wake. For quantitative analysis, profiles of $\bar{u}, \bar{v}$, $\overline{C_{p}}, \overline{u^{\prime} u^{\prime}}, \overline{v^{\prime} v^{\prime}}$ and $\overline{u^{\prime} v^{\prime}}$ are presented in figure $26(d-i)$. The profiles are obtained from the horizontal probe line in the near-wall region behind the slant in the $Z_{1}$ plane. It can be seen that the results suggest a high consistency of profiles between the fine and the medium meshes. However, large difference is found with the coarse mesh. Therefore, the medium mesh produces an ability comparable to that of the fine mesh for predictions of asymmetric 


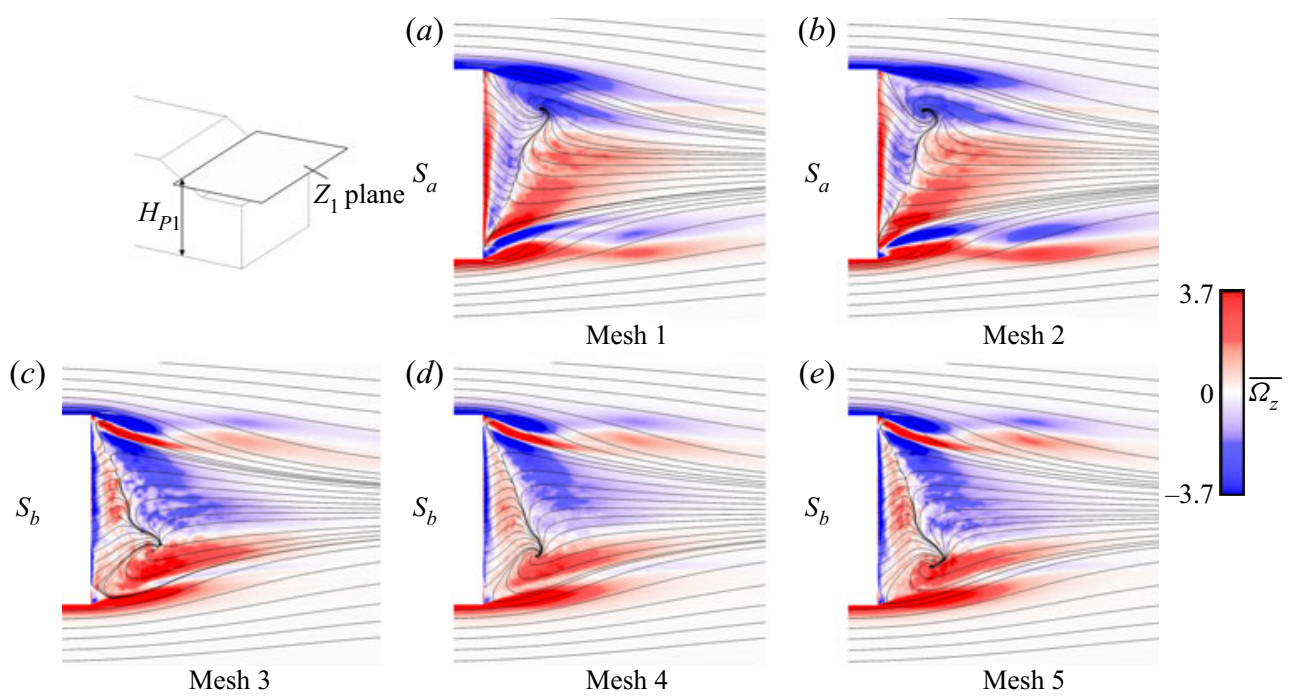

Figure 28. Mean streamlines and the distribution of vertical vorticity, $\overline{\Omega_{z}}$, projected on the $Z_{1}$ plane, obtained by using well-resolved meshes: (a) Mesh 1; (b) Mesh 2; (c) Mesh 3; (d) Mesh 4; (e) Mesh 5.

flows considering the velocity, pressure and second-order Reynolds stresses. The coarse mesh simulation is found to be under-resolved resulting in a false symmetric flow. Since the incorrect wake symmetry is simulated by the coarse mesh with the maximum $\Delta_{s}^{+}$of 100 arranged throughout the middle body, the prediction of asymmetric wakes depends on the streamwise resolution upstream of the wake. After many attempts to modify the coarse mesh, an appropriate decrease of $\Delta_{s}^{+}$on the middle body is found to trigger the symmetry breaking.

A quantitative assessment of the wake asymmetry identified by the base pressure gradients is presented in figure 27. The pressure gradients illustrate highly consistent PDF distributions obtained from the fine mesh (shown in red) and the medium mesh (shown in blue). The wake asymmetry is indicated in the concentration of the pressure gradients with negative or positive values on each surface. However, the symmetry of the flow simulated with the coarse mesh (shown in green) is indicated in the concentration of the base pressure gradients approaching 0 . Therefore, the coarse mesh proves incapable of correctly resulting in the expected wake asymmetry. As a compromise between accuracy and computing resources, the medium mesh showing sufficient prediction ability is selected for the present LES investigation.

\section{Appendix B. Asymmetric mirrored states of bi-stability}

Several well-resolved LES are performed for Model 1 under the same boundary conditions with the aim of capturing the random switching process. Five of them are shown in figure 28 , with the visualization of the mean streamlines and vertical vorticity behind the slant averaged during the asymmetric state. Meshes 1-3 have resolutions similar to that of the medium mesh. Mesh 4 is the medium mesh and Mesh 5 is the fine mesh. One of the two asymmetric states, $S_{a}$ or $S_{b}$, randomly occurs in these simulations after the physical establishment of the flow. Therefore, the wake behind Model 1 is confirmed to be bi-stable. The asymmetric states, $S_{a}$ and $S_{b}$, are classified as two mirrored states of the wake bi-stability. 


\section{K. He and others}

\section{REFERENCES}

Ahmed, S.R., Ramm, G. \& Faltin, G. 1984 Some salient features of the time-averaged ground vehicle wake. SAE Trans. 93, 473-503.

Aljure, D.E., Lehmkuhl, O., Rodríguez, I. \& Oliva, A. 2014 Flow and turbulent structures around simplified car models. Comput. Fluids 96, 122-135.

Barros, D., Borée, J., CADot, O., SpOHn, A. \& NoACK, B.R. 2017 Forcing symmetry exchanges and flow reversals in turbulent wakes. J. Fluid Mech. 829, R1.

Bonnavion, G., Cadot, O., Évrard, A., Herbert, V., Parpais, S., Vigneron, R. \& Délery, J. 2017 On multistabilities of real car's wake. J. Wind Engng Ind. Aerodyn. 164, 22-33.

CARR, G.W. 1974 Influence of rear body shape on the aerodynamic characteristics of saloon cars. MIRA Rep. 1974/2.

Cogotti, A.A. 1986 Car-wake imaging using a seven-hole probe. SAE Tech Paper. 860214.

Dalla Longa, L., Evstafyeva, O. \& Morgans, A.S. 2019 Simulations of the bi-modal wake past three-dimensional blunt bluff bodies. J. Fluid Mech. 866, 791-809.

Evrard, A., Cadot, O., Herbert, V., Ricot, D., Vigneron, R. \& Délery, J. 2016 Fluid force and symmetry breaking modes of a 3D bluff body with a base cavity. J. Fluids Struct. 61, 99-114.

Evstafyeva, O., Morgans, A.S. \& Dalla Longa, L. 2017 Simulation and feedback control of the Ahmed body flow exhibiting symmetry breaking behaviour. J. Fluid Mech. 817, R2.

Gaylard, A., Howell, J. \& GarRy, K. 2007 Observation of flow asymmetry over the rear of notchback vehicles. SAE Tech Paper 2007-01-0900.

Gilhome, B.R., Saunders, J.W. \& Sheridan, J. 2001 Time averaged and unsteady near-wake analysis of cars. SAE Tech Paper. 2001-01-1040.

Grandemange, M., Cadot, O. \& Gohlke, M. 2012 Reflectional symmetry breaking of the separated flow over three-dimensional bluff bodies. Phys. Rev. E 86 (3), 035302(R).

Grandemange, M., Gohlke, M. \& CAdot, O. $2013 a$ Turbulent wake past a three-dimensional blunt body. Part 1. Global modes and bi-stability. J. Fluid Mech. 722, 51-84.

Grandemange, M., Gohlke, M. \& CAdot, O. $2013 b$ Bi-stability in the turbulent wake past parallelepiped bodies with various aspect ratios and wall effects. Phys. Fluids 25 (9), 095103.

Haffner, Y., Borée, J., Spohn, A. \& CASTElain, T. 2020 Mechanics of bluff body drag reduction during transient near-wake reversals. J. Fluid Mech. 894, A14.

He, K., Minelli, G., Wang, J.B., GaO, G.J. \& Krajnović, S. 2021 Assessment of LES, IDDES and RANS approaches for prediction of wakes behind notchback road vehicles. J. Wind Engng Ind. Aerodyn. 217, 104737.

Hinterberger, C., Garcia-Villalba, M. \& Rodi, W. 2004 Large eddy simulation of flow around the Ahmed body. In The Aerodynamics of Heavy Vehicles: Trucks, Buses, and Trains, pp. 77-87. Springer.

JENKINS, L.N. 2000 An experimental investigation of the flow over the rear end of a notchback automobile configuration. SAE Tech Paper. 2000-01-0489.

Krajnović, S. \& DAVidson, L. 2003 Numerical study of the flow around a bus-shaped body. J. Fluid Engng 125 (3), 500-509.

KrAjnović, S. \& DAVidson, L. 2005 Flow around a simplified car, part 2: understanding the flow. J. Fluid Engng 127 (5), 919-928.

LAwson, N.J., GARry, K.P. \& FAucompret, N. 2007 An investigation of the flow characteristics in the bootdeck region of a scale model notchback saloon vehicle. Proc. Inst. Mech. Engrs. D: J. Automob. Engng 221 (6), 739-754.

Lucas, J.M., Cadot, O., Herbert, V., Parpais, S. \& Délery, J. 2017 A numerical investigation of the asymmetric wake mode of a squareback Ahmed body-effect of a base cavity. J. Fluid Mech. 831, 675-697.

Lumley, J.L. 1970 Stochastic Tools in Turbulence - Applied Mathematics and Mechanics, vol. 12. Academic Press.

Meile, W., Ladinek, T., Brenn, G., Reppenhagen, A. \& Fuchs, A. 2016 Non-symmetric bi-stable flow around the Ahmed body. Intl J. Heat Fluid Flow 57, 34-47.

Minelli, G., Krajnović, S., BasARA, B. \& NoACK, B.R. 2016 Numerical investigation of active flow control around a generic truck A-pillar. Flow Turbul. Combust. 97 (4), 1235-1254.

NiCOUd, F. \& DuCRos, F. 1999 Subgrid-scale stress modelling based on the square of the velocity gradient tensor. Flow Turbul. Combust. 62 (3), 183-200.

Östh, J., NoAck, B.R., Krajnović, S., BArros, D. \& Borée, J. 2014 On the need for a nonlinear subscale turbulence term in POD models as exemplified for a high-Reynolds-number flow over an Ahmed body. J. Fluid Mech. 747, 518-544.

PAsquetti, R. \& Peres, N. 2015 A penalty model of synthetic micro-jet actuator with application to the control of wake flows. Comput. Fluids 114, 203-217. 


\section{Numerical investigation of the wake bi-stability}

PAvia, G. \& PAssmore, M. 2017 Characterisation of wake bi-stability for a square-back geometry with rotating wheels. In FKFS Conference, pp. 93-109. Springer.

Pavia, G., Passmore, M.A., Varney, M. \& Hodgson, G. 2020 Salient three-dimensional features of the turbulent wake of a simplified square-back vehicle. J. Fluid Mech. 888, A33.

Rao, A., Minelli, G., Basara, B. \& Krajnović, S. 2018 On the two flow states in the wake of a hatchback Ahmed body. J. Wind Engng Ind. Aerodyn. 173, 262-278.

Roshko, A. 1993 Perspectives on bluff body aerodynamics. J. Wind Engng Ind. Aerodyn. 49 (1-3), 79-100.

Roumeas, M., Gillieron, P. \& Kourta, A. 2009 Analysis and control of the near-wake flow over a square-back geometry. Comput. Fluids 38 (1), 60-70.

Serre, E., Minguez, M., Pasquetti, R., Guilmineau, E., Deng, G.B., KornhaAs, M., Schäfer, M., Fröhlich, J., Hinterberger, C. \& Rodi, W. 2013 On simulating the turbulent flow around the Ahmed body: a French-German collaborative evaluation of LES and DES. Comput. Fluids 78, 10-23.

Sims-Williams, D.B. \& DunCAN, B.D. 2003 The Ahmed model unsteady wake: experimental and computational analyses. SAE Tech Paper. 2003-01-1315.

Sims-Williams, D., Marwood, D. \& Sprot, A. 2011 Links between notchback geometry, aerodynamic drag, flow asymmetry and unsteady wake structure. SAE Intl J. Passenger Cars Mech. Syst. 4 (1), 156-165.

SIROVICH, L. 1987 Turbulence and the dynamics of coherent structures. Part I: coherent structures. Q. Appl. Maths 45 (3), 561-571.

Thacker, A., Aubrun, S., Leroy, A. \& Devinant, P. 2013 Experimental characterization of flow unsteadiness in the centerline plane of an Ahmed body rear slant. Exp. Fluids 54 (3), 1479.

Volpe, R., Devinant, P. \& Kourta, A. 2015 Experimental characterization of the unsteady natural wake of the full-scale square back Ahmed body: flow bi-stability and spectral analysis. Exp. Fluids 56 (5), 99. 